\title{
New Insights into White-Light Flare Emission from Radiative-Hydrodynamic Modeling of a Chromospheric Condensation.
}

\author{
Adam F. Kowalski ${ }^{1,2}$. S. L. Hawley ${ }^{3}$. \\ M. Carlsson ${ }^{4}$. J. C. Allred ${ }^{2}$. \\ H. Uitenbroek ${ }^{5}$. R. A. Osten ${ }^{6}$. \\ G. Holman ${ }^{2}$. \\ (C) Springer $\bullet \bullet \bullet$
}

\footnotetext{
${ }^{1}$ Department of Astronomy, University of Maryland, College Park, MD 20742, USA. email: adam.f.kowalski@nasa.gov

${ }^{2}$ NASA Goddard Space Flight Center, Heliophysics Science Division, Code 671, 8800 Greenbelt Rd., Greenbelt, MD 20771, USA.

${ }^{3}$ Department of Astronomy, University of Washington, Box 351580, Seattle, WA 98195, USA.

${ }^{4}$ Institute of Theoretical Astrophysics, University of Oslo, P.O. Box 1029, Blindern, N-0315, Oslo, Norway.

${ }^{5}$ National Solar Observatory, Sacramento Peak, P.O. Box 62 Sunpsot, NM 88349, USA.

${ }^{6}$ Space Telescope Science Institute, 3700 San Martin Drive, Baltimore, MD 21218, USA.
}

Abstract The heating mechanism at high densities during M-dwarf flares is poorly understood. Spectra of M-dwarf flares in the optical and near-ultraviolet wavelength regimes have revealed three continuum components during the impulsive phase: 1) an energetically dominant blackbody component with a color temperature of $T \approx 10^{4} \mathrm{~K}$ in the blue-optical, 2) a smaller amount of Balmer continuum emission in the near-ultraviolet at $\lambda \leq 3646 \AA$ and 3) an apparent pseudo-continuum of blended high-order Balmer lines between $\lambda=3646 \AA$ and $\lambda \approx 3900 \AA$. These properties are not reproduced by models that employ a typical solar-type flare heating level of $\leq 10^{11} \mathrm{erg} \mathrm{cm}^{-2} \mathrm{~s}^{-1}$ in non-thermal electrons, and therefore our understanding of these spectra is limited to a phenomenological three-component interpretation. We present a new 1D radiative-hydrodynamic model of an M-dwarf flare from precipitating non-thermal electrons with a large energy flux of $10^{13} \mathrm{erg} \mathrm{cm}^{-2} \mathrm{~s}^{-1}$. The simulation produces bright nearultraviolet and optical continuum emission from a dense $\left(n>10^{15} \mathrm{~cm}^{-3}\right)$, hot $(T \approx 12000-13500 \mathrm{~K})$ chromospheric condensation. For the first time, the observed color temperature and Balmer jump ratio are produced self-consistently in a radiative-hydrodynamic flare model. We find that a $T \approx 10^{4} \mathrm{~K}$ blackbodylike continuum component and a small Balmer jump ratio result from optically thick Balmer $(\infty \rightarrow n=2)$ and Paschen recombination $(\infty \rightarrow n=3)$ radiation, 
and thus the properties of the flux spectrum are caused by blue $(\lambda \approx 4300 \AA)$ light escaping over a larger physical depth range compared to red $(\lambda \approx 6700 \AA)$ and near-ultraviolet $(\lambda \approx 3500 \AA)$ light. To model the near-ultraviolet pseudocontinuum previously attributed to overlapping Balmer lines, we include the extra Balmer continuum opacity from Landau-Zener transitions that result from merged, high order energy levels of hydrogen in a dense, partially ionized atmosphere. This reveals a new diagnostic of ambient charge density in the densest regions of the atmosphere that are heated during dMe and solar flares.

\section{Introduction}

It is notoriously difficult to explain the origin of heating at high atmospheric densities during solar and stellar flares. The broadband color (white-light) distribution of the ultraviolet and optical emission during large and small flares on chromospherically active M-dwarf (dMe) stars exhibits the general shape of a $T=8500-9500 \mathrm{~K}$ blackbody (Hawley and Fisher, 1992; Hawley et al., 2003) without an indication of a significant Balmer jump at $\lambda=3646 \AA$. A recent homogeneous analysis of flare spectra around the Balmer jump has confirmed the presence of a hot blackbody (or blackbody-like) component with a color temperature of $T \gtrsim 10,000 \mathrm{~K}$ that contributes most of the radiated near-ultraviolet (NUV) and optical energy in the impulsive phase (Hawley and Pettersen, 1991; Kowalski et al., 2013, hereafter, K13). Intriguingly, the presence of hot blackbody emission with $T \approx 9000 \mathrm{~K}$ has recently also been inferred from Sun-as-a-star observations during large (X-class) and small (C-class) solar flares (Kretzschmar, 2011). The hot (hereafter, "hot" refers to temperatures between 8500 and 15,000 $\mathrm{K}$ ) blackbody continuum component is often interpreted as evidence of impulsive heating in a region with a high density of $n_{e} \approx 10^{15} \mathrm{~cm}^{-3}$ or more, which has been inferred from phenomenological modeling of dMe flares (Cram and Woods, 1982; Houdebine, 1992; Christian et al., 2003). A recent observation that directly supports this idea is the Vega-like flare spectrum detected during the 2009 January 16, megaflare event on the dM4.5e star YZ CMi Kowalski et al., 2010, 2013), which indicates that photospheric-level densities can be produced and maintained at sufficiently high temperatures during dMe flares. However, heating high densities requires a large amount of volumetric energy deposition such that the heating per unit mass is non-negligible. Direct heating of the photosphere by non-thermal (NT) deka-keV electrons is problematic because the electrons experience rapid energy loss as soon as they impact the top of the "thick-target" chromosphere.

A possible mechanism for increasing the density in the upper chromosphere was proposed in the early gas dynamic simulations of Livshits et al. (1981). These authors showed that a shock from precipitating non-thermal electrons (with an energy flux of $10^{12} \mathrm{erg} \mathrm{cm}^{-2} \mathrm{~s}^{-1}$ ) produces a rapid downflow and compression of the material to a very high $\left(>10^{15} \mathrm{~cm}^{-3}\right)$ density. The development of downward moving "chromospheric condensations" (hereafter, CC) is accompanied by an upward moving "evaporation" of chromospheric material as it is heated beyond a million degrees (Fisher. Canfield, and McClvmont, 
1985). Chromospheric evaporation and CCs are thought to be the fundamental processes in solar and stellar flares driving the frequently observed Neupert effect (Dennis and Zarro, 1993; Hawley et al., 1995; ; Guedel et al., 1996). Several studies have explored the role of explosive CCs as the source of white-light emission in solar and dMe flares (Livshits et al., 1981: Gan et al., 1992: Katsova, Boiko, and Livshits, 1997), but the formation threshold of CCs is strongly dependent on the initial atmospheric structure, the energy distribution (power law index, low-energy cutoff, and flux) of the precipitating NT electrons (Fisher, 1989), and the time-dependent ionization of helium (Abbett and Hawlev, 1999: Allred et al., 2005, 2006), thus necessitating an accurate and realistic description of the NT electron distribution, the pre-flare atmosphere structure, and the level populations of important cooling agents.

Recent radiative-hydrodynamic (RHD) simulations of dMe (Allred et al., 2006) flares with the RADYN code (Carlsson and Stein, 1995, 1997) explored the atmospheric response to an actual NT electron spectrum that was obtained from the peak of an X-class solar flare inferred from Reuven Ramaty High Energy Solar Spectroscopic Imager (RHESSI) data (Holman et al., 2003). A constant, solartype flare heating level of the NT electrons was employed with injected energy fluxes of $10^{10} \mathrm{erg} \mathrm{cm}^{-2} \mathrm{~s}^{-1}$ (F10) and $10^{11} \mathrm{erg} \mathrm{cm}^{-2} \mathrm{~s}^{-1}$ (F11). These simulations produced explosive mass motions indicative of chromospheric evaporation and a $\mathrm{CC}$, but with densities far too low to produce strong, blackbody-like white-light emission. Moreover, the prominent spectral component in these simulations was a hydrogen recombination spectrum with strong Balmer recombination continuum emission and a smaller amount of Paschen recombination continuum emission. In these models, the heating at high density was achieved through Balmer and Paschen continuum backwarming of the photosphere, which increased in temperature by $1200 \mathrm{~K}$ in the F11 run and produced additional amounts of optical/red (blackbody-like) emission. For the range of non-thermal electron energy fluxes thus far explored in RHD models (F9-F11; see also Hawley and Fisher, 1994; Abbett and Hawley, 1999), a flare spectrum with hot blackbody emission has not been produced through the development of a CC, through photospheric heating from Balmer and Paschen continuum backwarming (Allred et al., 2006), or through photospheric heating from X-rav and EUV backwarming (Hawley and Fisher, 1992; Allred et al., 2006, see also the extensive comparison of RHD model predictions and recent observations in Section 9 of Kowalski et al. (2013)).

In addition to hot blackbody emission, the recent spectral observations of dMe flares also show evidence for a secondary continuum component in the NUV at $\lambda \lesssim 3700 \AA$. A jump in flux at these wavelengths is not accounted for by an extrapolation of a single hot blackbody component that can be fit to the redder wavelengths at $\lambda>4000 \AA$. The jump in flux relative to the blackbody flux is smaller in the peak phase than in the gradual decay phase, when the color temperature of the hot blackbody component also decreases. A comparison to the F11 RHD model spectra from Allred et al. (2006) led to the conclusion that Balmer recombination continuum emission was needed to account for the additional flux in the NUV (Kowalski et al., 2010). However, high resolution $(R \approx 40000)$ echelle flare spectra show that the NUV continuum from $\lambda \leq 3700$ $\AA$ can exhibit the color temperature of a hot blackbody $(\approx 11000 \mathrm{~K})$ while not 
exhibiting any signature of a Balmer discontinuity or edge feature (Fuhrmeister et al., 2008) that is indicative of Balmer recombination continuum emission. Instead, the highest order Balmer lines are broadened and appear to diminish as they converge into a "pseudo-continuum" at wavelengths between $\lambda=3646$ $\AA$ and the bluest identifiable Balmer line, which is typically H15 $\lambda 3712$ or H16 $\lambda 3704$ for both large solar and dMe flares (Donati-Falchi, Smaldone, and Falciani, 1984; Hawley and Pettersen, 1991; Kowalski et al., 2010).

The broadening and merging of the high order Balmer lines in flares is thought to be due to the well-known Stark effect from ambient charges (Svestka, 1963; Donati-Falchi, Falciani, and Smaldone, 1985). The Balmer edge itself is also thought to be broadened, but thermodynamically self-consistent (where the internal partition function converges) modeling of this effect was not available until the occupational probability theory of pressure ionization was developed by Hummer and Mihalas (1988). This improved description of pressure ionization from Stark broadening at the Balmer edge is currently employed in white dwarf atmospheric modeling codes (Tremblay and Bergeron, 2009), but has not yet been implemented in models of solar and stellar flares to determine if the theory can explain the NUV/blue continuum, and in particular, the lack of a Balmer edge. The Stark broadening of the higher order Balmer lines is potentially a useful tool for constraining the environment (i.e., charge density) where the (blackbody) continuum emission is produced in stellar flares (Cram and Woods, 1982).

What atmospheric conditions and atomic processes at high density produce a flare radiation field that self-consistently exhibits all the important continuum components and line properties in the blue and NUV? First, we must relate the observables in blue/NUV spectra to physical parameters of the atmosphere. Then, the spectra can be used as powerful tools to constrain the heating mechanisms that produce these atmospheric conditions. In this paper, we explore the possible role of a very dense CC for producing white-light emission during dMe flares, which reveals a meaningful interpretation of the spectra that will be used to constrain targeted modeling of individual solar and stellar flares. These simulations are timely due to new spectral constraints in the blue/NUV and optical wavelength regimes (Kowalski et al., 2013) and modern computational facilities enabling calculation of detailed spectra with sophisticated radiative transfer codes. Section 2 describes our method of solution and the starting atmospheric conditions, Section 3 describes the atmospheric dynamics and a new interpretation of the $10^{4} \mathrm{~K}$ blackbody color temperature, Section 4 describes the improved modeling of the Balmer edge, Section 5 explores the viability of the model, Section 6] summarizes the study and presents several conclusions, and Section 7 describes future modeling work.

\section{Method of Solution and Initial Atmosphere}

\subsection{NLTE Radiative-Hydrodynamic Modeling with RADYN}

We performed 1D radiative-hydrodynamic modeling using the RADYN code (Carlsson and Stein, 1992, 1994, 1995, 1997, 2002). RADYN solves the equations 
of mass, momentum, internal energy, non-equilibrium level populations, charge conservation, and radiative transfer (see also Abbett and Hawlev, 1999). The electron and ion temperatures are equal and an adaptive grid (Dorfi and Drury, 1987) with 250 grid points resolves shocks in the atmosphere. The NLTE (nonLTE) problem is solved in RADYN using the technique of Scharmer (1981) and Scharmer and Carlsson (1985). The NLTE level populations for three atoms are calculated: a hydrogen atom (six levels including $\mathrm{H}$ II), a helium atom (five levels for He I, three levels for He II, and He III), and a singly ionized calcium ion (six levels including the Ca III ground state), giving a total of 22 bound-bound (b-b) and 19 bound-free (b-f) transitions calculated in detail (see Allred et al., 2005). Continua from elements other than $\mathrm{H}, \mathrm{He}$, and $\mathrm{Ca}$ II are treated as background continua in LTE using the Uppsala atmospheres program (Gustafsson, 1973). A radiative cooling function from CHIANTI (Dere et al., 1997; Young et al., 2003) at 42 temperature points between $10^{4} \mathrm{~K}$ and $10^{8} \mathrm{~K}$ is included to account for optically thin cooling. Radiation from several ionization states (e.g., $\mathrm{Mg}$ II, Fe II, and Si II) that are likely optically thick at $10^{4} \mathrm{~K}$ (Hansteen, 2012, private communication) may be important in flares Avrett. Machado, and Kurucz, 1986) but is not yet included in the models (see Section 7 ).

Each line profile is calculated with up to 100 frequency points across the transition using a Voigt profile and complete redistribution. For Lyman transitions, the effects of partial redistribution are approximated by truncating the line profiles at ten Doppler widths. A change to the line broadening due to the Stark effect is implemented in RADYN compared to the models of Allred et al. (2006). Here, we use the analytic formulae (Method 1) from Sutton (1978) to add the Stark damping parameter to the total damping parameter in the Voigt profiles for hydrogen. The Stark broadening of calcium and helium transitions remains the same as in previous versions of the code.

Backwarming from coronal X-ray and extreme-ultraviolet (XEUV) radiation is treated according to the method of Hawley and Fisher (1994), Gan and Fang (1990), and Abbett and Hawley (1999), which approximates the radiation source using a plane-parallel geometry within $1 \mathrm{Mm}$ and as a point-source beyond this distance. The emissivity at 41 temperature points from $10^{4}-10^{8} \mathrm{~K}$ in 14 wavelength bins from $\lambda=1-2500 \AA$, is generated from the Astrophysical Plasma Emission Code (APEC; Smith et al., 2001). The hydrogen, helium, and metal cross sections are obtained from CHIANTI.

We ran flare simulations with the starting atmospheric parameters and boundary conditions similar to those used in Allred et al. (2006), in order to facilitate the comparison. A preflare, plane-parallel 1D atmosphere with $\log g=4.75$ was relaxed to have velocities $\lesssim 10 \mathrm{~m} \mathrm{~s}^{-1}$ and converged NLTE populations. The upper temperature boundary was initially fixed at $6 \times 10^{6} \mathrm{~K}\left(n_{e} \approx 2.6 \times 10^{10} \mathrm{~cm}^{-3}\right)$, and constant non-radiative heating was applied at column mass $m$ greater than $3.16 \mathrm{~g} \mathrm{~cm}^{-2}\left(\tau_{5000}=0.06\right)$ to emulate a convective energy flux. The atmosphere was relaxed using a plane-parallel approximation of the X-ray backwarming, as would be appropriate from extended overlying active region loops. The effective temperature of the relaxed atmosphere is $3630 \mathrm{~K}$, corresponding to that of an early to mid M-dwarf. The height coordinate $(z)$ of the atmosphere lies along the length of a semi-circular magnetic loop of half-length $L_{1 / 2}=10^{9} \mathrm{~cm}(10$ 
$\mathrm{Mm})$, with $z=0$ at the photosphere $\left(\tau_{5000}=1.0, \log m=1.14 \mathrm{~g} \mathrm{~cm}^{-2}\right)$. A modification to the gravitational acceleration is incorporated near the top of the loop (Abbett and Hawley, 1999); otherwise, the geometry is assumed to be a vertical tube of constant cross-sectional area. The imposed 1D geometry assumes the plasma motion is confined to the direction of the magnetic field, as would be appropriate for a low plasma $\beta$ (plasma $\beta=\frac{P_{\text {gas }}}{P_{\text {mag }}}$ ), but is not necessarily satisfied in the photosphere of the preflare atmosphere (the equipartition magnetic field strength is $4.2 \mathrm{kG}$ at the photosphere, consistent with observations (Johns-Krull and Valenti, 1996)) or in the chromosphere during the flare. Detailed molecular transitions that are important for flux redistribution in an M-dwarf photosphere have not yet been included in the opacity.

\subsection{Flare Heating Prescription}

We model the atmospheric heating from a double power law, non-thermal (hereafter, NT) electron beam injected at the top of the atmosphere. In this study, we seek to directly connect to the work of Allred et al. (2006), which used the thicktarget parameters inferred from RHESSI (Lin et al., 2002) hard X-ray spectra during the peak of the 2002 July 23 X4.8 GOES class solar flare (Holman et al. 2003, see also Ireland et al. (2013)). A double power law of electron energies is parameterized by $\delta_{l}=3.0$ (the power law index from the cutoff energy to the break energy), $\delta_{u}=4.0$ (the power law index at energies greater than the break energy), $E_{c}=37 \mathrm{keV}$ (the "cutoff energy" or lowest energy of the NT electron spectrum), and $E_{B}=105 \mathrm{keV}$ (the break energy between $\delta_{l}$ and $\delta_{u}$ ). The value of $E_{c}$ derived from observations is an upper limit, and $E_{c}=37 \mathrm{keV}$ is moderately large compared to typically assumed or inferred values on the order of $20 \mathrm{keV}$. Note also that the break in hard X-ray photon spectra (and thus also the inferred double power law shape of the NT electron spectrum) can be the result of the non-uniform ionization of the ambient atmosphere (Su. Holman. and Dennis, 2011) or from energy losses to the return-current electric field (Holman, 2012). The parameters of the NT electron spectrum are poorly constrained during dMe flares because the hard X-ray emission is too faint to be observed except during the largest flares (Osten et al., 2010). A powerful superflare on the RS CVn HR 1099 is consistent with a hard NT electron spectrum with a single power law index $\delta \approx 3$ (Osten et al., 2007), similar to the relatively hard double power law spectrum employed in our simulations.

Free parameters which may not be well-constrained by RHESSI observations alone are the energy flux (Krucker et al., 2011) and the precise time profile (duration) of the flare heating in any given magnetic loop. We extend the range of heating fluxes from Allred et al. (2006) to also consider those with $10^{12} \mathrm{erg}$ $\mathrm{cm}^{-2} \mathrm{~s}^{-1}$ (F12) and $10^{13} \mathrm{erg} \mathrm{cm}^{-2} \mathrm{~s}^{-1}(\mathrm{~F} 13)$, in addition to $10^{11} \mathrm{erg} \mathrm{cm}^{-2}$ $\mathrm{s}^{-1}$ (F11). The F13 flux is not much larger than the largest values that have been derived during solar flares (Neidig, Grosser, and Hrovat, 1994), and such a level has recently been suggested to be relevant to the brightest solar flare kernels (Krucker et al., 2011; Milligan et al., 2014; Gritsyk and Somov, 2014). The duration of the heating in a single magnetic loop in solar flares is constrained in some cases to $\leq 25-60 \mathrm{~s}$ (Wang. Fang, and Ding, 2007; ; Schrijuer et al., 2006) 
and in other cases may be several minutes (Qiu et al., 2010; Warren, 2006). A wavelet analysis of high-time resolution hard X-ray light curves for 647 solar flares observed with the Compton Gamma Ray Observatory (CGRO) indicates a range in the duration of individual bursts from $0.1 \mathrm{~s}$ to $50 \mathrm{~s}$ (Aschwanden et al., 1998). The shortest burst durations from high-time resolution constraints observed in the NUV in dMe flares range from $\approx 2 \mathrm{~s}$ (Robinson et al., 1995) to $10 \mathrm{~s}$ (Mathioudakis et al., 2006). For our heating profile, we use a constant energy flux for a duration of $2.3 \mathrm{~s}$, after which the NT electron energy deposition decreases to 0 , and the cooling phase is modeled up to $5.1 \mathrm{~s}$ for the F13 model (and $30 \mathrm{~s}$ for the models with lower beam fluxes). Short heating bursts represent the heating of a single loop, which can be superposed in sequence to emulate an arcade development as has been done for soft X-ray data of solar flares Warren, 2006).

The elastic collisions between NT electrons and the ambient charged and neutral components of the atmosphere heat the gas through Coulomb interactions. Following Hawley and Fisher (1994), Abbett and Hawley (1999), and Allred et al. (2005, 2006), the NT electron energy deposition rate is included as an extra term in the conservation of energy equation. The Coulomb heating rate as a function of height is modeled here using the analytic, non-relativistic test-particle approach of Emslie (1978), which was modified to include the height-dependent ionization fraction of the atmosphere in Hawley and Fisher (1994). This prescription is based on the collisional thick-target model (Brown, 1971), but it also includes an approximation for pitch angle scattering of the NT electrons (Emslie, 1978). The inelastic collisions between NT electrons and the ambient neutral hydrogen component of the atmosphere cause direct excitation and ionization (Mott and Massey, 1965); we follow Kašparová et al. (2009) and add these NT collisional rates to the thermal rates for hvdrogen according to the prescription in Fang, Hénoux, and Gan (1993) 1. The results from Dalgarno and Griffing (1958) are included in these rates to account for secondary ionizations of hydrogen by electrons that are ionized by the beam from the ground state of hydrogen.

\section{Properties of the NUV and Optical Continuum Predictions}

\subsection{Comparison to Observables}

Continuum predictions from radiative-transfer modeling are constrained with two simple observational parameters: 1) the Balmer jump ratio and 2) the color temperature of the continuum at wavelengths longer than the Balmer edge. These two parameters were referred to as $\chi_{\text {flare }}$ and $T_{\mathrm{BB}}$, respectively, in a recent homogeneous analysis of 20 flares on dMe stars from K13. A blackbody function is fit to the blue window (BW) wavelength ranges (see K13) of the flux spectrum

\footnotetext{
${ }^{1}$ A comprehensive study of NT rates was presented in the calculations of Ricchiazzi (1982) and Ricchiazzi and Canfield (1983), who found that the $\mathrm{H}$ I $n=1 \rightarrow \infty$ and $n=1 \rightarrow n=2$ rates are important, but that the $n=2 \rightarrow \infty$ rate was negligible compared to the $n=2 \rightarrow \infty$ thermal rates for their range of beam fluxes.
} 
from $\lambda=4000-4800 \AA$ (the blue-optical wavelength regime) to obtain $T_{\mathrm{BB}}$, while $\chi_{\text {flare }}$ is the ratio of the flux averaged over $30 \AA$ centered on $\lambda=3615$ $\AA$ (blueward of the Balmer jump) to the flux averaged over $30 \AA$ centered on $\lambda=4170 \AA$ (redward of the Balmer jump). These wavelength ranges are least affected by emission lines while also being near the Balmer jump (see K13). We note that the detected continuum emission shortward of the Balmer jump in K13 also included wavelengths between $\lambda=3420 \AA$ and $\lambda=3600 \AA$. A more secure detection and characterization of this continuum emission would include shorter wavelengths (such as in Heinzel and Kleint, 2014, for a solar flare observed from space), but the signal-to-noise decreases at $\lambda<3600 \AA$ due to the atmospheric cutoff. The distribution of these parameters was found to fall between $\chi_{\text {flare }}=1.5-4$ and $T_{\mathrm{BB}}=9000-14000 \mathrm{~K}$ during the peak phases of nearly all of the flares in the sample from K13. The value of $\chi_{\text {flare,peak }}$ varied with flare type, with the most impulsive flares exhibiting a narrow range of $1.5-2.2$ and the gradual flares exhibiting larger values. We measure these same parameters from the flux spectra for the peak phases of the model continua for the range of beam fluxes studied here. The model continua are calculated at a fixed number of points across the NUV and optical, and so we interpolate the continua to the wavelengths used in the observations. We also compute the ratio of the line-integrated, continuum-subtracted $\mathrm{H} \gamma$ emission line flux to the value of the continuum flux at $\lambda=4170 \AA$ (hereafter, $\mathrm{H} \gamma_{\mathrm{EW} 4170}$; note, this was referred to as $\mathrm{H} \gamma / \mathrm{C} 4170$ in $\mathrm{K} 13)$.

The model predictions for $\chi_{\mathrm{flare}}, T_{\mathrm{BB}}, \mathrm{H} \gamma_{\mathrm{EW} 4170}$ are given for each NT electron flux in Table 1. For the F13 simulation, the table gives the coarse time evolution of these parameters for the impulsive and gradual phases. The evolution of the flux spectrum for F13 is shown in Figure相. At early times, the F13 flux spectrum exhibits a large Balmer jump ratio and a cool color temperature in the blueoptical. However, from $t=1.2 \mathrm{~s}$ to $2.2 \mathrm{~s}$, the continuum at $\lambda>3646 \AA$ becomes bluer as the flux at $\lambda=4300 \AA$ increases significantly while the flux at $\lambda \approx 6690$ $\AA$ increases only marginally. This results in an increase in the blue-optical color temperature, $T_{\mathrm{BB}}$, of $2000 \mathrm{~K}$. Also, the Balmer jump ratio decreases from 3.3 to 2 as the flux at $\lambda \approx 3550 \AA$ decreases while the flux at $\lambda=4300$ increases. The continuum at wavelengths shorter than the Balmer edge also becomes bluer over this time interval as the flux at $\lambda<3000 \AA$ increases while the flux at $\lambda \approx 3550 \AA$ decreases. In Section 3.3, we will explain how these changes in the flux spectrum are related to the increasing hydrogen b-f opacities in the atmosphere.

At $t=2.2 \mathrm{~s}$, the F13 simulation produces a flux spectrum that falls into the regime of the observations for the peak phase of dMe flares with a relatively small Balmer jump ratio, $\chi_{\text {flare }}=2.0$, and a blue-optical color temperature of $T_{\mathrm{BB}}=9300 \mathrm{~K}$. The F13 model NUV flux between $\lambda=3000-3646 \AA$ also exhibits the color temperature of a hot blackbody with $T=11500 \mathrm{~K}$. Although the signal-to-noise of spectra at NUV wavelengths is lower than the data at blueoptical wavelengths (due to the atmospheric cutoff), the color temperature in the NUV is generally consistent with the value derived at blue-optical wavelengths

${ }^{2}$ The flux spectra for the F13 simulation are available upon request. 
(cf. Figures $8-9$ of K13). The $\mathrm{H} \gamma_{\mathrm{EW} 4170}$ also falls within the range of the observed values at flare peak ( $c f$. Figures $11-12$ of K13). Blue-optical emission with a color temperature higher than $\approx 6000 \mathrm{~K}$ has not previously been selfconsistently reproduced in RHD models of dMe flares (K13). The F11 and F12 simulations do not produce properties that are consistent with observations of the impulsive phase: the Balmer jump ratios are too large $(>7)$ and the color temperatures are too low $(<6000 \mathrm{~K})$. From Table 1, it can be seen that the time-evolution of the characteristics of the F13 simulation is consistent with the general time-evolution observed in dMe flares: the Balmer jump and $\mathrm{H} \gamma_{\mathrm{EW} 4170}$ decrease in the rise phase and increase in the decay phase. The timescale of the burst $(5 \mathrm{~s})$, however, is much shorter than the timescales of most dMe flares (minutes to hours). Adding up sequentially heated magnetic strands as in the spatial development of a flare arcade (e.g., Warren, 2006; Qiu et al., 2010) is outside the scope of this work, but we intend to pursue this in a future paper.

\subsection{Dynamics and Energy Balance in the F13 Atmosphere}

The evolution of the F13 flux spectrum in Figure 1 is tied to the dynamical evolution of the atmosphere. We discuss here general properties of the hydrodynamics.

The NT electrons initially deposit their energy over a broad extent, with significant volumetric heating from $275 \mathrm{~km}\left(\log m=-2.1 \mathrm{~g} \mathrm{~cm}^{-2}\right)$ to the top of the chromosphere at $450 \mathrm{~km}\left(\log m=-4.05 \mathrm{~g} \mathrm{~cm}^{-2}\right)$, and there is nonnegligible NT electron energy deposition (300 $\left.\mathrm{erg} \mathrm{s}^{-1} \mathrm{~cm}^{-3}\right)$ directly in the corona. The maximum energy deposition from the NT electrons $\left(8 \times 10^{5} \mathrm{erg} \mathrm{s}^{-1}\right.$ $\left.\mathrm{cm}^{-3}\right)$ initially occurs at $z_{o}=390 \mathrm{~km}\left(\log m=-3.05 \mathrm{~g} \mathrm{~cm}^{-2}\right)$, which is $99 \%$ neutral but with a significant electron density $n_{e}=10^{12} \mathrm{~cm}^{-3}$. Most of the NT energy is deposited initially into NT hydrogen ionization at $z_{o}$. As the electron density increases at $z_{o}$ an increasing fraction of the NT energy goes into raising the temperature since heating of electrons occurs more efficiently than heating of neutrals (note, the thermalization of electrons, protons, and neutrals is assumed to happen instantaneously in these models).

Hydrogen becomes completely ionized by $t=0.002 \mathrm{~s}$ and thermal ionization increases over NT ionization as the temperature exceeds $20000 \mathrm{~K}$. He I then becomes the primary cooling agent as the temperature reaches $40000 \mathrm{~K}$. At $t=0.003 \mathrm{~s}$, the temperature approaches $65000 \mathrm{~K}$ and most helium at this height is now singly ionized. From $t=0.003 \mathrm{~s}$ to $t=0.01 \mathrm{~s}$ the He III fraction increases from $2 \%$ to $99.6 \%$ at $z_{X}=425 \mathrm{~km}$ as the temperature rapidly increases to $140000 \mathrm{~K}$. At $t=0.016 \mathrm{sec}$, a localized temperature maximum of $180000 \mathrm{~K}$ forms at $z_{X}$, and the thermal pressure generates upward velocities of $4 \mathrm{~km} \mathrm{~s}^{-1}$ and downward velocities of $2 \mathrm{~km} \mathrm{~s}^{-1}$. These mass motions are the seeds to the explosive condensation and evaporation which follow from runaway heating as material is evacuated from this region of the atmosphere.

Meanwhile at $t=0.016 \mathrm{~s}$, between $z=450$ and $z=460 \mathrm{~km}$ (at the preflare transition region heights), there is a strong upward flow of $30 \mathrm{~km} \mathrm{~s}^{-1}$ resulting from the increase in temperature to $90000 \mathrm{~K}$ of lower density material (previously the upper chromospheric material) between the site of maximum beam 
heating $(425 \mathrm{~km})$ and the transition region $(450 \mathrm{~km})$. At $t=0.2 \mathrm{~s}$, the evaporation consists of two fronts: a high density chromospheric front with maximum velocity of $125 \mathrm{~km} \mathrm{~s}^{-1}$ and a low density transition region front with maximum velocity of $250 \mathrm{~km} \mathrm{~s}^{-1}$. The downward front is a high density "chromospheric condensation" (CC), with a maximum velocity of $95 \mathrm{~km} \mathrm{~s}^{-1}$. The maximum densities of the chromospheric fronts have increased from $n_{H} \approx 2 \times 10^{14} \mathrm{~cm}^{-3}$ $(t=0.2 \mathrm{~s})$ to $n_{H} \approx 5 \times 10^{14} \mathrm{~cm}^{-3}(t=0.4 \mathrm{~s})$ and the temperatures have decreased from $50000 \mathrm{~K}$ to $37000 \mathrm{~K}$. At the location of $z_{X}$, the temperature has reached nearly $10 \mathrm{MK}$ by $t=0.4 \mathrm{~s}$ from the runaway NT electron heating as the density has decreased (material is evacuated) from $n_{H}=5 \times 10^{13} \mathrm{~cm}^{-3}$ $(\mathrm{t}=0 \mathrm{~s})$ to $n_{H}=6 \times 10^{12} \mathrm{~cm}^{-3}(t=0.4 \mathrm{~s})$. At the upper and lower ends of the transition from very hot $(T \approx 400000 \mathrm{~K}-10 \mathrm{MK})$ plasma to $T \approx 35000 \mathrm{~K}$ plasma, upward and downward propagating shock fronts are located at the very steep (nearly discontinuous) jumps in gas pressure, where the He III fraction changes from $100 \%$ to $<30 \% 3$

At $t=0.33 \mathrm{~s}$, the timesteps of the simulation become unmanageably small $(\Delta t \approx 10-100 \mathrm{~ns})$ due to the precision requirements of RADYN in calculating the population levels of He I and He II in regions of strong temperature gradient, especially at the upper evaporation fronts. At $t=0.478 \mathrm{~s}$, we decrease the accuracy of the minor level populations, with accurate solutions provided only for level population densities that are $>10^{-9}$ of the total elemental population density (initially, the parameter is $10^{-12}$ ), while the second derivative of the grid weights is increased to weight more toward the higher density (CC) front. The result is a dramatic increase in the time step size until $t=5.1 \mathrm{~s}$ as the low-density (upper) chromospheric evaporation front is smoothed over (the number of grid points here drastically decreases and the temperature is no longer maintained at $35000-50000 \mathrm{~K})$. The results for the simulation at $z>450 \mathrm{~km}$ are thus less accurate, but this approximation is required in order to compute $1 \mathrm{D}$ simulations with very steep and dynamic temperature and density gradients. We focus only on the radiating material in the CC and below, since this higher density material produces most of the optical emission; the model above $450 \mathrm{~km}$ should not affect the analysis at the lower heights.

Figure 2 summarizes the dynamical evolution of the lower atmosphere at $t=2.2 \mathrm{~s}$, when the maximum white-light emission is present. The top panel shows the velocity of the downward moving CC. At this time in the CC, the maximum hydrogen density is $7.6 \times 10^{15} \mathrm{~cm}^{-3}$, or $17.4 \times 10^{-9} \mathrm{~g} \mathrm{~cm}^{-3}$, which is over a factor of 12 greater than the hydrogen density at this height $(z=255.4$ $\mathrm{km}$ ) before the flare. This mass density is comparable to the preflare density at $z \approx 145 \mathrm{~km}$, which lies between the temperature minimum and the lower chromosphere. The evolution of the speed and temperature of the maximum density zone in the $\mathrm{CC}$ is shown in the bottom panels. By $t=2.2 \mathrm{~s}$, the speed of the downflowing material has decreased to $\approx 50 \mathrm{~km} \mathrm{~s}^{-1}$ as it encounters denser material and higher gas pressure lower in the atmosphere, the equation of motion for which was derived analytically by Fisher (1989). Over the simulation, the ${ }^{3}$ The speed of sound in the $\mathrm{CC}$ at $t=0.4 \mathrm{~s}$ is $30 \mathrm{~km} \mathrm{~s}^{-1}$ whereas material is traveling at
speeds of nearly $100 \mathrm{~km} \mathrm{~s}^{-1}$. 
temperature of the region of maximum density in the $\mathrm{CC}$ decreases steadily; this is a result of larger hydrogen emissivity for lower temperatures while increasing the density in the $\mathrm{CC}$ gives a larger volumetric cooling rate. Eventually the temperature nearly settles between 12500 and $13000 \mathrm{~K}$ at $t=2.2 \mathrm{~s}$ as continuous injection of NT electrons deposit energy in the CC. After the NT electron energy deposition decreases to zero at $t=2.3 \mathrm{~s}$, the CC continues to propagate downward, increasing its density to $1.3 \times 10^{16} \mathrm{~cm}^{-3}$ at $t=4.0 \mathrm{~s}$. The temperature drops to $7000 \mathrm{~K}$ at this time (when the NT energy input is cut off), which is much larger than the preflare temperature of $\approx 5000 \mathrm{~K}$ at this height $(196.5 \mathrm{~km})$.

At $t=2.2 \mathrm{~s}$, the $E \approx 65-200 \mathrm{keV}$ NT electrons encounter a thick-target "wall" at the $\mathrm{CC}$ and produce a maximum energy deposition rate of $5 \times 10^{7}$ erg $\mathrm{cm}^{-3} \mathrm{~s}^{-1}$ (compared to a maximum of $<10^{6} \mathrm{erg} \mathrm{cm}^{-3} \mathrm{~s}^{-1}$ initially) at $z=256.9 \mathrm{~km}$; the full width at half maximum (FWHM) of the energy deposition rate is only $0.46 \mathrm{~km}$ (compared to $75 \mathrm{~km}$ initially). The simulation becomes computationally problematic shortly after this time and therefore the NT heating rate is cut off at $2.3 \mathrm{~s}$. The energy budget around the CC is shown in Figure 3 , which gives the individual contributions to the change in total internal energy per unit mass. Positive quantities contribute toward heating, excitation, and ionization; negative quantities contribute toward cooling, recombination, and de-excitation. The lower end of the CC from Figure 2 at $239.4 \mathrm{~km}$ is indicated by a vertical long-dashed line in Figure 3 In the CC, the NT electron energy deposition is balanced by the thin and thick (detailed) radiative losses, resulting in a net decrease in the internal energy (recombination and cooling). At deeper layers than the $\mathrm{CC}, E \gtrsim 200 \mathrm{keV}$ NT electrons can still penetrate and heat the atmosphere. At $z \approx 200 \mathrm{~km}$, there is net ionization and heating from both Balmer continuum backwarming (red diamonds) and NT electron energy deposition. At these depths, radiation from the optically thin loss function and from the detailed transitions does not counterbalance these sources of energy. There are also some contributions from compressional and viscous work where the CC impacts and "accretes" the stationary material below it.

3.3. Properties of the Contribution Function for Continuum Emission in the Flare Atmosphere

The CC decreases in temperature and increases in density over time (Figure 2). By $t=2.2 \mathrm{~s}$ the $\mathrm{CC}$ has attained temperatures $>12000 \mathrm{~K}$ and increased in density over 12 times the preflare density, thus producing a large amount of radiative cooling from hydrogen and other elements (Figure 31). In this section, we explore the height dependent properties of the emergent intensity from the $\mathrm{CC}$ at three continuum wavelengths calculated in detail: $\lambda=3550 \AA$ in the NUV, $\lambda=4300 \AA$ in the blue-optical, and $\lambda=6690 \AA$ in the red-optical, which are indicated in Figure 1. In Section 3.1 we found that the NUV and blue-optical F13 model peak spectrum (blue spectrum in Figure 1) is described by two blackbody spectra with color temperatures of $T=11,500 \mathrm{~K}$ between $\lambda=3000-3646 \AA$ and $T=9300 \mathrm{~K}$ at $\lambda>4000 \AA$ in relative amounts given by the value of the Balmer jump ratio $(\approx 2)$. In this section, we use the contribution 
function to the emergent intensity (Magain, 1986; Carlsson, 1998) to explain the origin and characteristics of the white-light continuum emission in this model spectrum. We use the following form of the contribution function, $C_{I}=d I_{\lambda} / d z$ (Leenaarts et al., 2013), for a plane-parallel atmospheres:

$$
C_{I}=\frac{d I_{\lambda}}{d z}=\frac{\chi_{\nu}}{\mu} S_{\nu} e^{-\tau_{\nu} / \mu} \frac{c}{\lambda^{2}}
$$

where $\chi_{\nu}$ is the height-dependent extinction coefficient (in units of $\mathrm{cm}^{-1}$; not to be confused with $\chi_{\text {flare }}$ the Balmer jump ratio), $\tau_{\nu}$ is the optical depth $\left(\int_{z}^{10^{9} \mathrm{~cm}} \chi_{\nu} d z\right)$, and $S_{\nu}$ is the height-dependent source function. More simply, this equation is $\frac{j_{\nu}}{\mu} e^{-\tau_{\nu} / \mu} \frac{c}{\lambda^{2}}$, or the plasma emissivity $\left(j_{\nu}\right)$ at a given height and frequency multiplied by the attenuation of the radiation (determined by $e^{-\tau_{\nu}}$ ) as it propagates outward in the direction of $\mu$ from that height 5 We discuss the atomic processes that contribute to the continuum emissivity in Section 3.4

In Figure 4, we show the contribution function to the emergent vertical intensity $(\mu \approx 1)$ for $\lambda=3550 \AA, 4300 \AA$, and $6690 \AA$ at $t=2.2 \mathrm{~s}$. The temperature profile (dashed line) of the flare atmosphere is qualitatively similar to that of the quiescent atmosphere, except that the flare chromosphere is hotter and has moved to higher column mass. Radiation escapes from two principal regions of the flare atmosphere: a stationary component (see Figure 21) produces a wide maximum in $C_{I}$ at $z \approx 200 \mathrm{~km}$ in the mid-/upper- flare chromosphere below the $\mathrm{CC}$, while the $\mathrm{CC}$ produces a very thin component peaking at $z \approx 256.3 \mathrm{~km}$ with maxima in $C_{I}$ of 950,140 , and $120 \mathrm{erg} \mathrm{cm}^{-2} \mathrm{~s}^{-1} \AA^{-1} \mathrm{sr}^{-1} \mathrm{~cm}^{-1}$ (extending above the top of the figure) for $\lambda=3550,4300$, and $6690 \AA$, respectively. Smaller values of $z$ (defined in Section 2) refer to larger physical depths, whereas larger values of $\Delta z$ refer to larger physical depth ranges in the atmosphere. We measure the FWHM of the narrow component of $C_{I}$ to compare the physical depth ranges $\left(\Delta z_{\mathrm{FWHM}}\right)$ over which radiation escapes from the CC. The $\Delta z_{\mathrm{FWHM}}$ is the largest for $\lambda=4300 \AA$ : the $\Delta z_{\mathrm{FWHM}}$ values are $1 \mathrm{~km}, 1.75 \mathrm{~km}$, and 1.25 $\mathrm{km}$ for $\lambda=3550,4300$, and $6690 \AA$, respectively. The contribution function in the stationary component of the flare atmosphere is also markedly different among these three wavelengths. Whereas $\lambda=4300 \AA$ has a local maximum at $200 \mathrm{~km}$, and $20-25 \%$ of the emergent intensity originates from layers below the $\mathrm{CC}$ at $z<230 \mathrm{~km}$, the other two wavelengths have very little emergent intensity originating from these depths, with $\lambda=3550 \AA$ showing the least. The arrows on the top of the figure indicate the layers at which $\tau_{\lambda}=1$ for each wavelength. The $\tau_{\lambda}=1$ layer occurs in the $\mathrm{CC}$ for $\lambda=3550$ and $6690 \AA$, and in the stationary flare layers for $\lambda=4300 \AA$. Therefore, the intensity at $\lambda=4300$

\footnotetext{
${ }^{4}$ The differential $d I_{\lambda} / d z$ in the definition of the contribution function is the same as the differential describing the variation of the specific intensity with height in the radiative transfer equation; however, these differentials are not equivalent.

${ }^{5} j_{\nu}$ is often used to describe the emissivity in units of photons $\mathrm{cm}^{-3} \mathrm{~s}^{-1} \mathrm{sr}^{-1} \mathrm{~Hz}^{-1}$ whereas $\eta_{\nu}$ is used for the emissivity in units of ergs $\mathrm{cm}^{-3} \mathrm{~s}^{-1} \mathrm{sr}^{-1} \mathrm{~Hz}^{-1}$; here, we use $j_{\nu}$ to describe the emissivity in units of ergs $\mathrm{cm}^{-3} \mathrm{~s}^{-1} \mathrm{sr}^{-1} \mathrm{~Hz}^{-1}$.
} 
$\AA$ originates over the largest physical depth range (largest $\Delta z$ ) and from the lowest atmospheric heights due to the smallest optical depth in the CC.

At times earlier than $2.2 \mathrm{~s}$, a larger fraction of the emergent intensity at $\lambda=3550 \AA$ and $6690 \AA$ originates from the stationary component of the flare atmosphere because the $\mathrm{CC}$ has a lower density and optical depth. The evolution of the optical depth for $\lambda=4300 \AA$ and $3550 \AA$ and the evolution of the column densities $(N)$ for hydrogen in $n=2$ and $n=3$ in the CC (where $v<-5 \mathrm{~km} \mathrm{~s}^{-1}$ ) are shown in Figure 5 As the $\mathrm{CC}$ becomes denser and attains temperatures that excite hydrogen to $n=2$ and $n=3$, the probability of photoionization by light with these wavelengths increases. The values of the column densities at $t=2.2 \mathrm{~s}$ of $N_{H}(n=2)>3 \times 10^{17} \mathrm{~cm}^{-2}$ and $N_{H}(n=3)>10^{17} \mathrm{~cm}^{-2}$ define useful requirement 6 for large optical depths $\left(\tau_{4300}=0.8, \tau_{3550}=5\right)$ in any future flare model of CCs with material near temperatures of $12000 \mathrm{~K}$.

The evolution of the contribution function results from increasing, wavelengthdependent optical depths at optical/NUV wavelengths and can explain the evolution of the flux spectrum in Figure 1 (Section 3.1). The very large and continuously increasing optical depth at $\lambda=3550 \AA$ causes a decrease in the flux at this wavelength between $t=1.2 \mathrm{~s}$ and $t=2.2 \mathrm{~s}$ because the radiation can only escape from a very thin layer. At $t=2.2 \mathrm{~s}$, the radiation at $\lambda=4300$ $\AA$ is still relatively optically thin in the $\mathrm{CC}(\tau<0.8)$, and therefore has a higher probability of escape, giving rise to a larger FWHM of the contribution function in the $\mathrm{CC}\left(\Delta z_{\mathrm{FWHM}}\right)$ and also a larger fraction that can escape from the stationary component of the flare atmosphere. The flux at $4300 \AA$ increases from $t=1.2 \mathrm{~s}$ to $2.2 \mathrm{~s}$ in Figure 1 and results from the increasing density but a lower optical depth due to the lower photoionization cross-section and smaller population density for $n=3$ compared to $n=2$. The larger optical depth at $\lambda=6690 \AA$ relative to $\lambda=4300 \AA$ is caused by a larger photoionization crosssection (but same population density), which leads to a much smaller increase in flux at this wavelength in Figure 1from $t=1.2-2.2 \mathrm{~s}$. The NUV flux significantly increases at $\lambda<3000 \AA$ and the color temperature at $\lambda=3000-3646 \AA$ becomes larger over this time interval due to similar reasoning for the increase of the flux at $\lambda=4300 \AA$ and the increase of the color temperature at $\lambda>4000 \AA$. Higher signal-to-noise flare spectra at $\lambda<3600 \AA$ would help to rigorously test the slope changes predicted by these models at NUV wavelengths.

\subsection{Opacity Components in the Optical Depth}

\subsubsection{The Optically Thick Hydrogen Recombination Flare Spectrum for the F13 model}

In this section, we quantitatively demonstrate how the properties of the flux spectrum at $t=2.2 \mathrm{~s}$ in Figure 1 primarily arise from spontaneous hydrogen recombination continuum emission by reconstructing the contribution function

${ }^{6}$ See Section 3.4 there are additional opacities from photoionization of the higher levels of hydrogen, hydrogen free-free absorption, and opacity from the $\mathrm{H}^{-}$ion that can also contribute towards the optical depth at these wavelengths. 
to the emergent intensity from atomic emissivity processes. The b-f opacity $\left(\chi_{\nu}\right)$ corrected for stimulated emission is given by Mihalas (1978, Equation 7-1):

$$
\chi_{\nu}=\Sigma_{i}\left\{\left[n_{i}-n_{i}^{*} e^{-h \nu / k T}\right] \sigma_{i}(\nu)\right\}
$$

where $\sigma_{i}$ is the photoionization cross-section of an atom with an electron in level $i$ for light of frequency $\nu ; n_{i}^{*}=n_{c} \times\left(n_{i} / n_{c}\right)^{*}$ where $n_{c}$ is the number density of protons and $\left(n_{i} / n_{c}\right)^{*}$ is the density ratio in LTE which can be calculated using the Saha equation. We calculate the hydrogen b-f and hydrogen free-free (f-f, bremsstrahlung) opacity at $\lambda=3550 \AA, 4300 \AA$, and $6690 \AA$ for the flare atmosphere at $t=2.2 \mathrm{~s}$. The total hydrogen continuum (b-f $+\mathrm{f}-\mathrm{f}$ ) opacity values for $\lambda=4300$ and $3550 \AA$ are shown in the top left of Figure 6 . The hydrogen b-f opacity dominates for both wavelengths, but the hydrogen $\mathrm{f}-\mathrm{f}$ is non-negligible in the CC at optical wavelengths. At $\lambda=4300 \AA$, the Paschen b- $\mathrm{f}(n=3 \rightarrow \infty)$ continuum opacity is largest, whereas at $\lambda=3550 \AA$ the Balmer b- $\mathrm{f}(n=2 \rightarrow \infty)$ continuum opacity dominates. The b-f and f-f opacities from the $\mathrm{H}^{-}$ion are not shown explicitly in Figure 6 but are included in RADYN and in the contribution function (Figure 4). The $\mathrm{H}^{-}$opacity is small $(\approx 3.5 \%)$ compared to the hydrogen b-f and f-f opacities in the CC but dominates at the lower temperatures $(T<$ $9000 \mathrm{~K})$ in the flare atmosphere at $z \lesssim 130 \mathrm{~km}$. Although the electron density is high in the CC, Thomson scattering is relatively very small.

Most of the CC is so dense that the LTE condition $S=B$ (where $B$ is the Planck function) applies and the source function is only dependent on local quantities. The level populations at $t=2.2 \mathrm{~s}$ for $n=2$ and $n=3$ are equal to the LTE populations, and collisional rates are generally large compared to radiative rates, for most of the flare atmosphere $(z=100-256.6 \mathrm{~km})$ except at the surface of the CC. The level population $n_{p}$ is the LTE value through all of the CC. The hydrogen b-f and f-f continuum emissivity, $j_{\lambda}$, is shown in the top right panel of Figure 6. For simplicity, only the spontaneous recombination emission is shown. The relative rates of induced to spontaneous recombination are very small at these wavelengths but can become larger at redder wavelengths. The hydrogen emissivity can be approximated as $\left\{\chi_{\nu, b-f}+\chi_{\nu, f-f}\right\} B_{\nu} \frac{c}{\lambda^{2}}$ through most of the CC; but to account for departures from LTE at the surface of the CC, we use the NLTE hydrogen b-f emissivity from Equation 7-2 of Mihalas (1978). The ratio of emissivities $\left(j_{\lambda=3550} / j_{\lambda=4300}\right)$ in the $\mathrm{CC}$ is about 9.5 , as we expect for hydrogen recombination (Balmer and Paschen) continuum emission at these temperatures. When $\tau$ is non-negligible, the wavelength dependent attenuation becomes important in the contribution function (Equation (1D). The attenuation factor, $e^{-\tau_{\lambda}}$, is shown in the bottom left panel of Figure 6. and illustrates how the $\lambda=3550 \AA$ emissivity is preferentially attenuated at $z=180-200 \mathrm{~km}$, where $\tau_{3550}=20$. Here, $\tau_{4300} \approx 1$ which is large enough to attenuate some of the radiation from these heights (compare the shift in peaks at this height indicated by vertical dashed lines in the top right and bottom right panels). The $e^{-\tau_{\lambda}}$ factor gives rise to the wavelength-dependent changes in the FWHM of $C_{I}$ $\left(\Delta z_{\mathrm{FWHM}}\right)$ and in the fraction of the emergent intensity that originates from the stationary component (Section 3.3 and Figure 4). 
The contribution function, $C_{I}$, is shown again in the bottom right panel of Figure 6 (note logarithmic y-scale as compared to Figure 4 which is on a linear scale). Integrating $C_{I}$ over height gives the emergent intensity at $\mu=0.95$. The integration over height reveals that the Balmer jump intensity ratio $\left(I_{\lambda=3550} / I_{\lambda=4300}\right.$, similar to $\chi_{\text {flare }}$ ) is $\approx 2.2$, a value indicative of the small Balmer jump in emission in the flux spectrum in Figure 1. The emergent optical intensity ratio $I_{\lambda=4300} / I_{\lambda=6690}$ of 2.3 is a value consistent with the color temperature of a hot $(\approx 9000 \mathrm{~K})$ blackbody. We have thus shown that hydrogen recombination (and bremsstrahlung) continuum emissivity between $11000-14000 \mathrm{~K}$ integrated over heights where optical depths become large $\left(\tau_{4300} \gtrsim 0.5\right)$ and are wavelength dependent (from the hydrogen cross-section dependence and thermal population density variation) can self-consistently generate the color temperature $\left(T_{\mathrm{BB}}\right)$ of the blue-optical emission and the Balmer jump ratio $\left(\chi_{\text {flare }}\right)$ in agreement with observations of the peak phases of dMe flares.

\subsubsection{The Optically Thin Hydrogen Recombination Flare Spectrum for the F11 model}

The contribution functions for the F11 model at $t=2.2 \mathrm{~s}$ are shown in Figure 7 $(\mathrm{a}-\mathrm{b})$. At this time, the atmosphere has not yet experienced explosive evaporation, which is consistent with the F11 simulation from Allred et al. (2006). In contrast to the F13 simulation, the $C_{I}$ profiles for $\lambda=3550 \AA$ and $4300 \AA$ during the F11 simulation extend over a large portion of the flare chromosphere. The $C_{I}$ profiles also have second peaks near the preflare photosphere, which is not shown here because the important molecular species and opacities in M-dwarf photospheres have not yet been included in RADYN (Section 2). The optical depths for $4300 \AA$ and $3550 \AA$ do not reach values of 1 until heights near the pre-flare photosphere; the values of $\tau_{3550}$ in the flare chromosphere range between 0.001 and 0.1 , with $\tau_{4300}$ about an order of magnitude less. Therefore, the NUV and optical radiation from the flare chromosphere is dominated by optically thin hydrogen Balmer and Paschen continua emission from recombination due to the increased electron density (and temperature). The emergent F11 NUV and optical spectrum is a combination of mostly unattenuated hydrogen recombination emission from $z=250-400 \mathrm{~km}$, combined with any increased photospheric radiation from backwarming (Allred et al., 2006). The value of $\chi_{\text {flare }}$ is large $\left(\approx 9\right.$; Table 1) and $T_{\mathrm{BB}}$ is low $(\approx 5300 \mathrm{~K}$; Table 1), as would be expected for hydrogen recombination at $T \approx 10000 \mathrm{~K}$ with negligible wavelength dependent attenuation.

If we use the formula for spontaneous thermal hydrogen recombination emissivity (Equation 7-2 of Mihalas, 1978, see also Aller (1963) and Wiese, Kelleher, and Paquette (1972)) in Equation (1), we obtain an approximate formula for the ratio of the contribution function at two wavelengths, $\lambda_{1}$ and $\lambda_{2}$ :

$$
\frac{C_{I}\left(\lambda_{1}\right)}{C_{I}\left(\lambda_{2}\right)} \approx \frac{\lambda_{2}^{2} n_{2}^{3} g_{\mathrm{bf}, 1}}{\lambda_{1}^{2} n_{1}^{3} g_{\mathrm{bf}, 2}} e^{\frac{h c}{k_{B} T_{e}}\left(\frac{1}{\lambda_{2}}-\frac{1}{\lambda_{1}}\right)} e^{1.578 \times 10^{5} / T_{e}\left(\frac{1}{n_{1}^{2}}-\frac{1}{n_{2}^{2}}\right)} e^{\left(\tau_{2}-\tau_{1}\right) / \mu}
$$

at height $z$ with $\left(T_{e}, n_{e}\right)$, where $g_{\mathrm{bf}}$ are the Gaunt factors and $n_{1}$ and $n_{2}$ are the principal quantum numbers that dominate the opacity in Equation (2) for 
$\lambda_{1}$ and $\lambda_{2}$, respectively7. When $\tau_{1,2}$ are negligible (as for the F11 in the flare chromosphere), $C_{I}\left(\lambda_{1}=4300\right) / C_{I}\left(\lambda_{2}=6690\right)$ for $\mu=1$ is $\approx 0.8$ at $T=10000$ $\mathrm{K}$ and $e^{\tau_{2}-\tau_{1}}$ is not much larger than 1 , which causes an optically thin Paschen recombination continuum to exhibit a color temperature of $\approx 5000 \mathrm{~K}$ Kunkel, 1970; Kerr and Fletcher, 2014, ; Section 9 of K13). When $\tau_{1,2}$ are non-negligible (as for the F13 at $t=2.2 \mathrm{~s}$, Section 3.4.1), the ratio of the attenuation factors is $e^{\tau_{2}-\tau_{1}}>1$ which causes the optical continuum to appear hotter. Therefore, increasing the optical depth makes the Paschen continuum bluer with a higher value of $T_{\mathrm{BB}}$ (apparently hotter), as can be seen by approximating the atmosphere as a slab with constant $\left(T_{e}, n_{e}\right)$ and integrating over height. Note, the correction of the b-f opacity for stimulated emission (second term in Equation (2)) also affects the color temperature because it decreases the opacity at $\lambda=4300 \AA$ by a smaller factor than at $\lambda=6690 \AA$. However, the actual emergent intensity is an integration over height with varying $\left(T_{e}, n_{e}\right)$, which is why we model the atmosphere gradients, which are apparent in Figure 2. The emergent radiative flux is a Gaussian integration of intensity over five $\mu$ values: the intensity at a smaller value of $\mu$ gives an even bluer optical continuum and smaller Balmer jump ratio due to the $e^{\left(\tau_{2}-\tau_{1}\right) / \mu}$ factor.

\section{Stark Broadening at the Balmer Edge}

Stark broadening is thought to be an important symmetric broadening mechanism of the hydrogen Balmer lines during solar and stellar flares (Svestka, 1963; Worden et al., 1984; Hawlev and Pettersen, 1991; Johns-Krull et al., 1997; Paulson et al., 2006: Allred et al.. 2006), in addition to possible turbulent (Eason et al., 1992; Doyle et al., 1988) and thermal contributions. The linear (first order) Stark effect is the splitting of the degenerate orbital angular momentum $(l)$ states of each principal quantum state $(n)$ of hydrogen, due to a net electric microfield from the surrounding distribution of charges. To date, the most complete theories of the linear Stark effect are those of Vidal. Cooper, and Smith (1970, 1971. 1973). Seaton (1990). Stehle and Jacquemot (1993), and Kepple and Griem (1968), which take into account the perturbations from the slowly moving (quasi-static) protons and from the rapidly moving (dynamic) electrons. The Vidal et al. treatment is known as the unified theory because it provides an accurate treatment of the electron perturbations from the line core through the far wings. Theories of Stark broadening typically only present results for the lowest order Balmer transitions, but Lemke (1997) extended the results from the Vidal et al. unified theory to $n=22$. Therefore, this Vidal et al. unified theory (which is considered the best treatment of the non-overlapping far line wings and of the higher order Balmer lines; see the discussion in Johns-Krull et al., 1997, and in Vidal. Cooper, and Smith (1970) ) is most useful to the interpretation of flare spectra in the Balmer jump region 8 .

${ }^{7}$ The value $1.578 \times 10^{5}$ in the second exponential is $13.598 \mathrm{eV} / k_{B}$.

Bengtson. Tannich, and Kepple $(1970)$ provided results up to H12 using the theory of Kepple and Griem (1968) for one temperature and electron density. Bengtson (1996, private communi- 
In future work (Section 7), we will implement the Lemke (1997) extension of the Vidal et al. results (see Section 7). In RADYN, the analytic approximations of the Stark damping from Sutton (1978) are added to the total damping in the Voigt profile (Section 2). Solar modeling of infrared hydrogen lines has shown that this is adequate (Carlsson and Rutten, 1992).

\subsection{Occupational Probability of Level $n$}

Here, we address the atomic physics necessary to interpret the merging of the rapidly converging higher order Balmer lines and the apparent lack of a Balmer discontinuity (or edge) at $\lambda=3646 \AA$ in flare spectra.

To model the Stark broadening of the Balmer edge, we apply the occupational probability theory developed in Hummer and Mihalas (1988) and Dappen, Anderson, and Mihalas (1987), which is summarized thoroughly in the recent modeling study of cool DA white-dwarf atmospheres of Tremblay and Bergeron (2009). This theory provides a thermodynamically self-consistent approach to pressure ionization, which allows the internal partition function to converge. Unlike procedures that truncate the internal partition function, occupational probability theory includes a continuous transition from bound to free states as upper levels of hydrogen converge and Stark energy shifts cause them to overlap. Electric field ionization experiments with sodium have shown that enhanced ionization rates of electrons in level $n$ occur when there is a critical electric microfield strength causing the highest Stark state of $n$ to cross with the lowest Stark state of $n+1$ (Pillet et al., 1984). At a level crossing, electrons can undergo a Landau-Zener transition from $n$ to $n+1$ as the electric field fluctuates with time above and then below the critical value (Zener, 1932). For electric field perturbations that cause level crossing of $n$ and $n+1$, all states $\geq n$ become dissolved or destroyed, and an electron excited to $n$ can undergo a reverse-cascade to the continuum through a series of Landau-Zener transitions.

The occupational probability, $w_{n}$, is the probability of an atom being perturbed by a net electric microfield that is below the critical field strength that would dissolve/destroy level $n$ :

$$
w_{n}(\text { charged })=\int_{0}^{\beta_{\text {crit }}} \mathrm{P}(\beta) \mathrm{d} \beta
$$

where $P(\beta)$ is the probability distribution of net electric microfield strength $(\beta)$ and $\beta_{\text {crit }}$ is the critical net electric microfield that dissolves/destroys level $n$ (Seaton, 1990). Both the high frequency electron collisions and quasi-static proton collisions contribute to level dissolution, but $w_{n}$ is primarily determined from the ambient protons (Hummer and Mihalas, 1988; Tremblay and Bergeron, 2009). The ambient electrons contribute to the cascade plus ionization process through their high-frequency collisions with the electrons that are excited to the

cation) provided higher order line profiles for the study of Johns-Krull et al. (1997), but these results have not been made publicly available to our knowledge. 
dissolved states and through the mixing of Stark states allowing the reversecascade of Landau-Zener transitions to proceed (see Tremblay and Bergeron, 2009). Following Tremblav and Bergeron (2009), we adopt the $Q$-fit electric microfield model (Navfonov et al., 1999) of the Hooper distribution to represent the probability distribution $P(\beta)$. In our calculations, we use the total occupational probability obtained by multiplying Equation (4) by the probability of level destruction from neutral collisions, which are modeled using the same method described in Tremblay and Bergeron (2009) and Hummer and Mihalas (1988). For the conditions in flare atmospheres, the total occupational probability is approximately equal to $w_{n}$ (charged), given by Equation (4).

\subsection{Continuous Opacities at the b-f Edge}

Dappen, Anderson, and Mihalas (1987) developed the method for incorporating the occupational probability theory into b-f and b-b opacities for hydrogen to account for the effects of Landau-Zener transitions and the subsequent cascade to the continuum.

The Landau-Zener b-b opacity for a Balmer line, $\mathrm{H} n$, is the nominal b-b opacity multiplied by the occupational probability $w_{n} / w_{n=2}$, where $w_{2}=1$ for the conditions in flare atmospheres. The Landau-Zener b-f opacity is the nominal b-f opacity (Equation (2)) extrapolated to wavelengths longer than the standard b-f edge $\left(\lambda_{o}\right)$, multiplied by the probability that an atom experiences a critical microfield strength or greater, causing the upper level of the transition to be dissolved. For any wavelength $\lambda>\lambda_{o}$, this probability is obtained by using a "pseudo" quantum number $n^{*}$ :

$$
n^{*}=\left(\frac{1}{n_{i}^{2}}-\frac{h c}{\lambda Z^{2} E}\right)^{-1 / 2}
$$

where $E=13.598 \mathrm{eV}=2.1787102 \times 10^{-11} \mathrm{erg}$ and $Z$ is the atomic number ( $Z=1$ for hydrogen). The dissolved probability (or dissolved fraction) is

$$
D(\lambda)=1-\frac{w_{n^{*}}}{w_{i}}
$$

where $w_{n *}$ is the occupational probability for level $n^{*}$ and $i=2$ for the Balmer lines. As discussed by Dappen, Anderson, and Mihalas (1987) the pseudo levels $\left(n^{*}\right)$ are simple extrapolation tools between the limits of $w_{n} \approx 1$ and $w_{n}<<1$ that are useful for estimating the critical microfield strength (where the highest pseudo-stark state of $n^{*}$ and lowest of $n^{*}+1$ overlap) that can cause level dissolution through a cascade of Landau-Zener transitions. Also, note, there are several different ways to estimate the critical field strength, and we use the popular method from Seaton (1990).

Now accounting for Landau-Zener transitions, wavelengths longer than the Balmer edge $\left(\lambda_{\mathrm{o}}=3646 \AA\right)$ allow there to be a fraction of atoms in $n=2$ that can be photoionized by $\lambda>3646 \AA$, whereas without Landau-Zener transitions, photons with $\lambda>3646 \AA$ could photoionize atoms only with electrons in $n>2$. 
The total b-f opacity, with and without Landau-Zener transitions, in a representative layer of the $\mathrm{CC}$ in the F13 model is shown in Figure 8, Due to the high ambient proton density $\left(5.6 \times 10^{15} \mathrm{~cm}^{-3}\right)$, the dissolved fraction is $>99 \%$ (the occupational probability, $w_{n}$, is $<1 \%$ ) for the upper levels that contribute opacity at $\lambda \lesssim 3730 \AA$. The nearly complete level dissolution results in a direct extrapolation of the Balmer b-f opacity to these wavelengths. As $\lambda$ increases, the fraction of $n=2$ hydrogen atoms that can be photoionized decreases, due to the larger critical microfield required to cause destruction of the upper level of an atomic transition. This leads to a continuous transition from the Balmer continuum opacity into the Paschen continuum opacity, which dominates at $\lambda>4200$ $\AA$. The hydrogen f-f opacity (without Landau-Zener transitions) is also shown in this figure, illustrating that it is small but non-negligible $\left(\chi_{\mathrm{f}-\mathrm{f}} / \chi_{\mathrm{tot}} \approx 0.2\right)$ at redder wavelengths.

Hereafter, we refer to these new opacities as Landau-Zener opacities, and the pseudo-continuum resulting from this opacity as the Landau-Zener continuum. Note, Dappen, Anderson, and Mihalas (1987) referred to this as the "dissolved level pseudo-continuum." In Figure9 we show how the Landau-Zener b-f opacity from $\lambda=3646 \AA$ to $\lambda=4200 \AA$ changes as a function of $n_{p}\left(\approx n_{e}\right)$. Selfconsistent atmospheric parameters were obtained from representative heights in the F11 and F13 models, at various times, and covering a range of proton densities, $n_{p}=10^{13}-5 \times 10^{15} \mathrm{~cm}^{-3}$. As the ambient charge density increases, the Landau-Zener b-f opacity maximum shifts from $\lambda=3660 \AA$ to $\lambda=3730 \AA$. Also shown here are the occupational probabilities, $w_{n} / w_{2}$ (open circles, right axis, $w_{2}=1$ ), that are multiplied by the nominal b-b opacity for each of the Balmer b-b transitions. Thus the opacity from the lines is transferred to the Landau-Zener continuum.

\subsection{Landau-Zener Opacity Modeling Using the RH Code}

We use the NLTE RH code (Uitenbroek, 2001) to model the Landau-Zener b-f and b-b opacities and emissivities between $\lambda=3646 \AA$ and $\lambda=5000 \AA$ for the F13 model at $t=2.2 \mathrm{~s}$. The RH code is well-suited for modeling the Balmer jump region due to the ability to calculate overlapping transitions (Rybicki and Hummer, 1992) with more wavelength points than in the original dynamic (RADYN) simulation. By assuming statistical equilibrium and hydrogen number conservation, we can use an arbitrarily large hydrogen atom to model the merging of higher order lines near the Balmer b-f edge. Statistical equilibrium is a poor assumption during the early phases of the flare simulation, but at $t=2.2 \mathrm{~s}$, the resulting error is small9. As in RADYN, the linear Stark broadening in RH is treated with a Voigt profile and the approximate formulae from Sutton (1978) are used; a prescription for quadratic Stark broadening is also included.

We obtain the atmospheric parameters (column mass, temperature, electron density, and velocity) at $t=2.2 \mathrm{~s}$ from the RADYN simulation with a sixlevel hydrogen atom, and we calculate the spectrum with the RH code using

${ }^{9}$ We compared the RH and RADYN predictions at $t=2.2 \mathrm{~s}$ for the Balmer lines and continua that overlap, and we found satisfactory agreement. 
a 20-level hydrogen atom. In the RH calculation, Ca II and He are treated as background (LTE) elements. At each depth in the atmosphere, the effect of Landau-Zener transitions is modeled by multiplying the Balmer b-f cross section by the dissolved fraction (Equation (6) ) and the Balmer b-b line profile by the occupational probability $\left(w_{n} / w_{2}\right)$, as in Tremblay and Bergeron (2009). The NLTE opacity and emissivity is then calculated in $\mathrm{RH}$ with these modifications. We show the result of the RH flux calculation in Figure 10, compared to several observed flare spectra during the mid rise $(\mathrm{S} \# 27) \sqrt{10}$, late rise $(\mathrm{S} \# 28)$, and peak phases (S\#31) of a large flare on the dM4.5e star YZ CMi from K13. The observed spectra have been normalized to the flux at $\lambda=4700 \AA$ to show the range of properties at $\lambda<3900 \AA$ and the lack of color temperature evolution at $\lambda>4000 \AA$ (see K13 for the evolution of the flare flux without scaling). Because the flare area is unresolved, the continuum brightness is not constrained by observations. Thus, the RH model surface-flux spectrum has been scaled by the factor $R_{\text {flare }}^{2} / d^{2}$ (where $R_{\text {flare }}$ is the projected radius of a circular flare area, and $d$ is the distance to the star) to match the $\lambda=4700 \AA$ flux of each observed flare spectrum. An instrumental convolution with a Gaussian of FWHM $=13.5 \AA$ has also been applied to the model. We are not attempting to model this flare here, but we use these spectra to illustrate that the model spectrum characteristics are similar to the data at least at some phases of the flare evolution.

First, the blue-optical color temperature between the Balmer lines at $\lambda>$ $4000 \AA$ is well fit to a blackbody with a temperature of $T_{\mathrm{BB}}=10400 \mathrm{~K}$, which is shown in Figure 10 as the dashed line. Note that this color temperature is larger by $1000 \mathrm{~K}$ compared to that in Table 1, due to the extension of the Landau-Zener continuum and Balmer wings between $\lambda=4000 \AA$ and $\lambda=4060 \AA 11$. Although the color temperature is represented by a blackbody with $T=10400 \mathrm{~K}$, the $\mathrm{F} 13$ model radiative flux is larger by a factor of 1.7 compared to the surface flux of a blackbody with this temperature. The radiation temperature $\left(T_{\mathrm{rad}, \mu=1, \lambda=4300} \approx 11600 \mathrm{~K}\right)$ is also significantly larger than the color temperature $\left(T_{\mathrm{BB}}=9300-10400 \mathrm{~K}\right)$. Hereafter, we refer to the hot blackbody component as the hot "blackbody-like" continuum component, since we have identified the emission as predominantly due to optically thick hydrogen (Paschen) recombination emission in Section 3.4.1. Second, a jump in flux occurs above an extrapolation of the blackbody to the bluest wavelengths, and this jump in flux is the same in both the F13 model and the observations of the late rise phase spectrum. Third, there is no Balmer discontinuity feature present in the model spectrum at $\lambda=3646 \AA$ : the model calculation produces a featureless Landau-Zener continuum connecting the Balmer continuum emission at $\lambda<3646 \AA$ to the bluest significant Balmer line in emission, H10 $\lambda 3798$. This effect originates from the direct extrapolation of Balmer continuum opacity in Figure 8 to these wavelengths where the opacity is due to the upper levels of hydrogen that have nearly $100 \%$ probability of being dissolved.

The RH flux spectrum with the opacity effects from Landau-Zener transitions (and without instrumental convolution) is shown in Figure 11 around the Balmer

\footnotetext{
${ }^{10}$ The S\#'s refer to the time-sequential spectrum numbers over each flare analyzed in K13.

${ }^{11}$ We use the same continuum blue window (BW) wavelength regions and weighting as in K13.
} 
edge region; no Balmer lines are at all apparent between $\lambda=3646-3740 \AA$, and $\mathrm{H} 11$ is barely identifiable. The RH prediction using a 20-level hydrogen atom without the effects from Landau-Zener transitions (gray line, "no L-Z") is shown and definitively illustrates that the overlap/merging of the Balmer wings indeed form a pseudo-continuum between the Balmer lines at wavelengths shorter than $4100 \AA$, but less than the calculation with the opacity effects from Landau-Zener transitions (black line, "L-Z"). The two calculations disagree most concerning the appearance and nature of the pseudo-continuum between $3646 \AA$ and $3720 \AA$. In particular, the "no L-Z" calculation exhibits a "blue continuum bump" at $\lambda \approx 3720 \AA$ and a large positive slope at wavelengths between 3646 $\AA$ and $3720 \AA$, whereas the "L-Z" calculation predicts a continuum with the same slope as the observed Balmer continuum at all $\lambda<3720 \AA$. The "no L-Z" calculation also shows that the H10-H19 lines do not merge enough to conceal the Balmer discontinuity, which is just as apparent if we apply a large instrumental broadening. Using a hydrogen atom with more levels (we also tried a 35-level hydrogen atom) does not improve the modeling result: the opacity effects from Landau-Zener transitions are necessary to completely conceal the Balmer edge at these densities. As discussed by Dappen, Anderson, and Mihalas (1987), the slope of the pseudo-continuum in the "no L-Z" calculation between $3646 \AA$ and the blue continuum bump is the result of the rapidly decreasing oscillator strength density of the higher-order transitions. In the "L-Z" calculation, Stark broadening decreases the oscillator strength density of the b-b transitions while replacing oscillator strength density with the Landau-Zener continuum.

In Figure 12, we show how the contribution function at $\lambda=3870 \AA$ (between H8 $\lambda 3889$ and H9 $\lambda 3835$ ) and $\lambda=4300 \AA$ compare with and without opacity effects from Landau-Zener transitions. As expected, the contribution at $\lambda=4300$ $\AA$ does not change. For $\lambda=3870 \AA$ without Landau-Zener transitions, the opacity is dominated by Paschen b-f opacity. This wavelength is more optically thin than $\lambda=4300 \AA$. Hence there is more contribution from the stationary component of the flare atmosphere at $z=150-230 \mathrm{~km}$. In the case with Landau-Zener transitions, the Landau-Zener opacity increases the optical depth beyond that of the optical depth at $4300 \AA$ (the contribution at $z=150-230$ $\mathrm{km}$ is less than in the calculation without Landau-Zener transitions) and more $\lambda=3870 \AA$ photons escape from higher in the atmosphere in the CC (at $z=251$ $\mathrm{km}$ ) due to recombination of electrons from the $n^{*}=8.3$ pseudo-level (Equation (5)) to the $n=2$ level of hydrogen.

We can now associate the model flux spectrum in Figure 10 to several atomic emission processes. At $\lambda<3646 \AA$ the flux is almost entirely Balmer continuum emission from free electrons recombining to $n=2$. This part of the spectrum also exhibits a color temperature $T_{\mathrm{BB}}>10000 \mathrm{~K}$, with a peak in the NUV between $\lambda=2500-2600 \AA$. The Landau-Zener continuum flux between $\lambda=3646-3760$ $\AA$ is bona-fide Balmer continuum recombination radiation resulting from free electrons recombining from the $n^{*}$ pseudo (dissolved) upper levels of hydrogen to $n=2$ (Dappen, Anderson, and Mihalas, 1987); i.e., undergoing reverse LandauZener transitions from the continuum to $n=n^{*}$, at which point they transition to $n=2$. The flux between the rapidly converging Balmer lines from $\mathrm{H} 10$ to $\mathrm{H} \epsilon$ is mostly Landau-Zener continuum emission from electrons recombining from 
the dissolved upper levels to $n=2$, but at these wavelengths there is a smaller fraction of atoms in the atmosphere that experience a critical field strength, and thus the Landau-Zener continuum emission is less than at $\lambda=3646-3760 \AA$. There is also some contribution from the far wings of the Balmer b-b transitions. The far wings of $\mathrm{H} \delta$ and $\mathrm{H} \epsilon$ and the redmost extent of the Landau-Zener continuum emission produce additional emission above the nominal Paschen recombination spectrum as red as $\lambda=4200 \AA$ (compare solid and dotted red lines). At $\lambda>4200 \AA$, Paschen continuum emission from recombination of free electrons to $n=3$ dominates, with a smaller $(\approx 20 \%)$ contribution from thermal bremsstrahlung (free-free) emission. Paschen recombination radiation also contributes toward the radiation at $\lambda<4200 \AA$ but to a much smaller degree than the calculation without including the Landau-Zener continuum, as can be seen by the attenuation of radiation at $z=160-230 \mathrm{~km}$ in Figure 12. An interesting observational effect of including the Landau-Zener continuum emission is that the measured color temperature of the flux spectrum increases from $9300 \mathrm{~K}$ (6 level, without Landau-Zener transitions, dotted red line, Table 1) to $10400 \mathrm{~K}$ (20 level, with Landau-Zener transitions, thick red line), improving further the applicability of the F13 model to the measured $T_{\mathrm{BB}}$ distribution at peak for a variety of flares (K13).

We note that the lower order Balmer lines $(\mathrm{H} \alpha-\mathrm{H} \delta)$ can be used to constrain the models. The CC in the F13 model generates broad profiles, large surface fluxes, and a reverse decrement (more $\mathrm{H} \gamma$ flux compared to $\mathrm{H} \alpha$ flux) in the lower order Balmer lines. The large widths of the lower order Balmer lines in Figure 10 result from Stark broadening. However, improvements to the implementation of the Stark effect (see Section 17) are required for a detailed comparison. Although the F13 model produces approximately 18 times the surface flux in $\mathrm{H} \gamma$ compared to the F11 model (and approximately 6 times that in the F12 model), the ratio of Balmer line to continuum values is notably lower than in the smaller NT electron flux simulations (Table 1). At $t=2.2 \mathrm{~s}$, the relative amount of Balmer line to continuum emission produced by the $\mathrm{CC}$ is consistent with typical observed values in the impulsive phase: $\mathrm{H} \gamma_{\mathrm{EW} 4170} \approx 20$ in the F13 model compared to $\mathrm{H} \gamma_{\mathrm{EW} 4170} \approx 15-30$ in the late rise and peak phase spectra in Figure 10, Additional flaring areas that are either impulsively heated with smaller NT electron flux or gradually decaying from a large electron flux may be required to more accurately reproduce the time evolution of the emission lines in these individual spectra.

\subsection{A New Diagnostic of Ambient Charge Density in Chromospheric Flares}

The opacity effects from Landau-Zener transitions result in Balmer recombination radiation longward of $3646 \AA$ and explain the NUV/blue "pseudo-continuum" previously attributed to the merging of Stark-broadened Balmer lines. The occupational probability theory as employed in white-dwarf atmospheric models provides a self-consistent quantum mechanical method of modeling the opacity effects from cascading Landau-Zener transitions. This new modeling explains the lack of an observed Balmer discontinuity (or edge) in flare spectra, while still allowing a Balmer jump in flux above an extrapolation of a blackbody 
that is fit to the blue-optical wavelengths in the Paschen continuum $(\lambda>4000$ $\AA)$. Accounting for Landau-Zener transitions gives an emergent flux spectrum from the F13 model at $t=2.2 \mathrm{~s}$ with continuum emission bridging Balmer continuum emission at $\lambda \leq 3646 \AA$ to the highest-order Balmer line that is detectable, which is $\mathrm{H} 10$ or H11 in the case of the F13 model. The microscopic picture that is reinforced here is that slowly moving thermal electrons are able to recombine to $n=2$ and produce a photon with wavelength $>3646 \AA$ (a Balmer continuum photon) due to the nearly complete dissolution of the upper levels: $1-w_{n} / w_{2} \geq 0.85$ for levels $n \geq 11$, in a dense plasma with $n_{p}=5 \times 10^{15} \mathrm{~cm}^{-3}$. The success of modeling the "pseudo-continuum" emission from $\lambda=3646-3760$ $\AA$ in Figure 10 as a red wavelength extension of Balmer continuum emission is consistent with our conclusion that the white-light emission, in this RHD model, is dominated by a combination of hydrogen Balmer b-f, hydrogen Paschen b-f, and hydrogen f-f emission.

In solar flares, a "pseudo-continuum" component between the Balmer lines from $\approx 3700-3870 \AA$ was first noted by Zirin $(1980)$ and Zirin and Neidig (1981). The current, best treatment of the modeling of the Balmer jump region and pseudo-continuum was applied to several solar flares by DonatiFalchi, Falciani, and Smaldone (1985), who used static, isothermal models with $n_{e}=10^{13.2}-10^{13.6} \mathrm{~cm}^{-3}$ and $T_{e}=7000-10,000 \mathrm{~K}$. These models produced a "blue continuum bump" at $\lambda=3675 \AA$, which shifts to redder wavelengths and increases in brightness for higher electron density, and has been discussed in light of a new solar flare observation with broad wavelength coverage spectra in the NUV (Kowalski. Cauzzi, and Fletcher, 2015). The pseudo-continuum bump at $\lambda \approx 3720 \AA$ in Figure 11 produced in our calculation without Landau-Zener opacity effects (Section 4.3) is very likely analogous to the NUV/blue bump feature at $\lambda \approx 3675 \AA$ produced in the Donati-Falchi, Falciani, and Smaldone (1985) lower density slab models. As noted by these authors, this feature is due to the merging of Stark broadened high order Balmer lines. Another feature in the Donati-Falchi. Falciani. and Smaldone (1985) models is a continuum "dip" at $3646 \AA$ due to the smearing of the Balmer edge. Donati-Falchi, Falciani, and Smaldone (1985) truncated the model hydrogen atom at $n=84$ and broadened the Balmer edge using the Stark width of the highest Balmer line. For $n=84$, the Stark damping parameter is $30 \AA$ (Svestka, 1963; Johns-Krull et al., 1997) which results in a flattening and smearing of the edge in their models. Since the Landau-Zener continuum modeling from occupational probability theory does not predict a continuum bump at $\lambda \approx 3720 \AA$ or a dip near the Balmer edge (for very high proton densities), this modeling may also provide a more selfconsistent explanation for the range in Balmer jump characteristics observed in solar flare spectra (as in the compilation by Neidig, 1983), while also improving the diagnostic capabilities of these spectra.

Intuitively, occupational probability theory provides insight beyond a model that considers pseudo-continuum from merged lines as just a superposition of b-b opacities. In the classical framework (Donati-Falchi, Falciani, and Smaldone, 1985), the opacity of a b-b transition is spread over a larger spectral range by the Stark broadening, and is then added to the opacity of other Stark-broadened b-b opacities at a given wavelength. In the occupational probability framework, 
the Stark-broadened b-b opacity is replaced by a larger b-f opacity at a given wavelength when hydrogen atoms experience a critical microfield perturbation. The larger b-f opacity results from the fact that free states are not degenerate (in orbital angular momentum), and therefore the oscillator strength density of the continuum is not re-distributed over a superposition of Stark states as for b-b transitions. This physical difference explains the large discrepancy between the two model spectra in Figure 11 from $\lambda=3646-3730 \AA$ (see also the discussion in Dappen. Anderson, and Mihalas, 1987). In summary, the opacity sources from Donati-Falchi. Falciani, and Smaldone (1985) are continuum opacity from a Stark-broadened b-f edge and a superposition of Stark-broadened $b$ - $b$ opacities whereas in occupational probability theory, the opacity sources are continuum opacity from Stark-dissolved levels and a superposition of the remaining undissolved Stark-broadened b-b opacities.

Modeling the opacity effects from Landau-Zener transitions provides a new physical interpretation of several dMe flare spectra in the literature. We've already shown how this modeling with a very large density $\left(\approx 5 \times 10^{15} \mathrm{~cm}^{-3}\right)$ can account for the continuum and line properties in a flare with H10 or H11 as the bluest identifiable Balmer line (Figure 10). In the continuum peak (1:15 UT) spectrum from the dMe flare studied in García-Alvarez et al. (2002), H9 or H10 also appear as the bluest Balmer line; however, their gasdynamic modeling using the Jevremovic et al. (1998) fit to the Balmer decrement indicates densities nearly two orders of magnitude lower than the occupational probability modeling indicates based on the bluest identifiable Balmer line. The bluest identifiable Balmer lines in most dMe flares with moderate spectral resolution are typically H15 and H16 both in the impulsive phase (Hawley and Pettersen, 1991) and in the gradual decay phase (Kowalski et al., 2010, 2013). Even $R=40000$ echelle spectra of dMe flares sometimes do not show emission in Balmer lines bluer than about H15 and instead exhibit a nearly featureless 12 continuum with a color temperature of $9000-11000 \mathrm{~K}$ at $\lambda \lesssim 3700 \AA$ (Figures 2 and 5 of Fuhrmeister et al., 2008). It is also notable that one peak flare spectrum of a dMe flare showed Balmer lines as blue as H19 (Fuhrmeister et al., 2011), which the authors concluded was a result of only a small enhancement of the chromospheric electron density in the flare. Since the Landau-Zener continuum resulting from occupational probability theory can fully account for similar characteristics of this spectral region in the flare spectra in Figure 10, we suggest the range of characteristics observed around the Balmer jump in stellar flares may be explained with the range of Landau-Zener opacities in Figure 9.

Future modeling of particular flares using occupational probability theory can be used to infer the magnitude and gradient of the ambient charge (proton) density in the densest regions of the flare atmosphere that are significantly heated and produce white-light emission. In Section 4.3 and Figure 9 , we showed how the Landau-Zener continuum opacity maximum changes and occupational probabilities dissolve the hydrogen Balmer transitions over 2.5 orders of magnitude in charge density. We present a simple method using the Balmer decrement to

\footnotetext{
${ }^{12}$ Aside from many faint metallic and helium emission lines.
} 
estimate the charge density for an optically thick flare atmosphere in LTE (as for the F13 atmosphere studied here). We introduce the Landau-Zener Balmer decrement, which is the flux in a Balmer line transition (e.g., H10 or H11 which are relatively unaffected by strong metallic or helium lines) that experiences a large change in the dissolved fraction of the upper level of this transition, divided by the flux of a lower order line that is not affected by level dissolution (e.g., $\mathrm{H} \gamma / 13$. In Table 2. we show how the Landau-Zener Balmer decrement $\mathrm{H} 11 / \mathrm{H} \gamma$ changes for different temperatures and over the density range of $n_{p}=10^{13}$ to $n_{p}=5 \times 10^{15} \mathrm{~cm}^{-3}$. This Balmer decrement is obtained by multiplying $w_{n=11} / w_{n=5}$ (where $w_{5} \approx 1$ ) by the Balmer decrement formula for the optically thick, isothermal, LTE limit in Appendix B of Drake and Ulrich (1980). The decrement sharply drops for $n_{p}>10^{15} \mathrm{~cm}^{-3}$ as $n=11$ becomes dissolved (see also Figure 91). The observed decrements for $\mathrm{H} 11 / \mathrm{H} \gamma$ in the two peak spectra from Hawley and Pettersen (1991) are 0.26 and 0.22 , which suggests a charge density of not much larger than $10^{15} \mathrm{~cm}^{-3}$ but also not smaller than $10^{14} \mathrm{~cm}^{-3}$. This method could be used as an alternative to the Inglis-Teller relation 14 , which requires data with high spectral resolution. However, since lines and continua are formed at different physical depths and depth ranges (as for the F13 atmosphere studied here), we plan to improve the accuracy of this spectral diagnostic using a grid of NLTE flare atmospheres in future works.

\section{The Viability of the F13 Model}

M-dwarf flares are more energetic than solar flares (in total). Typical white-light energies of dMe flares are $10^{30}-10^{32} \mathrm{erg}$ (Hawlev et al., 2014) with occasional events exceeding $10^{34} \mathrm{erg}$ in the $U$-band alone (Kowalski et al., 2010). The areal extent of flare footpoints cannot be measured directly 15 , and hard X-ray emission is far too faint except during the largest dMe flares (Osten et al., 2010). Although we cannot determine if NT electron fluxes on M-dwarfs are indeed as high as $10^{13} \mathrm{erg} \mathrm{cm}^{-2} \mathrm{~s}^{-1}$, typical values of $\approx 10^{11} \mathrm{erg} \mathrm{cm}^{-2} \mathrm{~s}^{-1}$ in strong solar flares cannot explain the properties of white-light emission during dMe flares, and thus we infer that the energy flux is higher. The atmospheric response to an F13 NT electron burst provides insight into the radiative transfer processes that could explain white-light emission, but is this very high flux a realistic model for $\mathrm{dMe}$ flares? In this section, we evaluate its viability and conclude that some issues still remain.

First, is an F13 beam even possible during an M-dwarf flare? The energy flux available to accelerate particles at the reconnection site is limited by the free

\footnotetext{
${ }^{13}$ Assuming that the Balmer line emission does not originate at a different spatial location, for example from a larger area with a lower density, than the continuum emission.

${ }^{14}$ See the discussion in Hummer and Mihalas (1988) about how the theory of occupational probability differs from the Inglis-Teller relationship: the Landau-Zener transitions require fluctuations of the microfield around the critical field value.

${ }^{15}$ Emission model fits, e.g., with a blackbody function or RHD model spectra, give an indirect estimate of the flare area and energy flux requirements.
} 
magnetic energy in the corona, which can be estimated by $\frac{B_{\text {cor }}^{2}}{8 \pi} v_{\text {inflow }}=2 \times 10^{13}$ erg $\mathrm{cm}^{-2} \mathrm{~s}^{-1}$, where $v_{\text {inflow }}$ is the rate at which magnetic field flows into the reconnection region and may be between $0.01-0.1$ times the Alfvén velocity. The energy flux requirement of $2 \times 10^{13} \mathrm{erg} \mathrm{cm}^{-2} \mathrm{~s}^{-1}$ into each magnetic footpoint assumes equipartition of energy among protons and electrons and that all free magnetic energy goes into accelerating particles. Using the larger estimate for inflow velocity, $0.1 \times v_{\text {Alfven }}$ gives $B_{\text {cor }} \approx 1.5 \mathrm{kG}$ as the coronal magnetic field (assuming $v_{\text {inflow }}=0.01 v_{\text {Alfven }}$ gives $3.3 \mathrm{kG}$ as the coronal magnetic field) necessary to supply this energy flux. Direct (Zeeman broadening) measurements of small-scale magnetic fields of M-dwarfs reveal average photospheric magnetic flux density of $3-4.5 \mathrm{kG}$ (Johns-Krull and Valenti, 1996; Phan-Bao et al., 2009), but the coronal magnetic field above these starspots has not been directly measured. The most well-studied active corona is that of the dM3.5e star EV Lac (Osten et al., 2006), which exhibits a very hot (10 MK), confined $\left(L \approx 10^{8} \mathrm{~cm}\right)$, and dense $\left(n_{e} \approx 10^{13} \mathrm{~cm}^{-3}\right)$ component in quiescence. For these structures, the minimum magnetic field required for confinement is estimated to be $\approx 1 \mathrm{kG}$. Other values from microwave data of flares and quiescence have suggested coronal magnetic fields on M-dwarfs exceeding $1 \mathrm{kG}$ (Osten et al., 2006; Smith, Güdel, and Audard, 2005), which have also been inferred from magnetic confinement estimates using the Haisch Simplified Analysis of data from the Extreme Ultraviolet Explorer (EUVE) of many M-dwarf flares (Mullan et al., 2006). Thus, the energy requirement for accelerating an F13 NT electron flux requires relatively high (kilo-Gauss) coronal magnetic fields but not outside the realm of current measurements.

Although the energy flux requirements may be plausible (given large Mdwarf coronal magnetic fields), the electron number flux for the F13 model may be problematic due to return-current effects. The electron number flux corresponding to our double power law F13 spectrum is $10^{20}$ electrons $\mathrm{cm}^{-2}$ $\mathrm{s}^{-1}$. A return-current electric field is established from the charge displacement of electrons and protons, given that both particles are energized equally in the process of magnetic reconnection and retraction. The initial energy lost per NT electron from the return-current electric field is $13 \mathrm{keV} \mathrm{Mm}^{-1}$ at the apex of our model loop (Equation 29 of Holman, 2012). The NT electrons with the cutoff energy $(37 \mathrm{keV})$ lose all of their energy and are thermalized after penetrating only $3 \mathrm{Mm}$ into the atmosphere, after which the flux of ongoing NT electrons decreases with distance as more NT electrons are thermalized. Holman (2012) derives the evolution of the NT electron flux distribution accounting for losses by the return-current electric field. We use these results and find that only NT electrons with $E_{\text {initial }}>85 \mathrm{keV}$ penetrate to the top of the chromosphere without thermalizing. Furthermore, the F13 NT electron density is comparable to the ambient density $\left(n_{e}=2.6 \times 10^{10} \mathrm{~cm}^{-3}\right)$ at the apex of our model coronal loop; this condition would cause the ambient electrons to be accelerated and would result in instabilities and other effects that are not modeled here.

A more basic issue is that the number flux of electrons available in the corona flowing into the reconnection site is only $\approx 5 \times 10^{18}$ electrons $\mathrm{cm}^{-2} \mathrm{~s}^{-1}$ (using $n_{e} \times 0.1 v_{\text {Alfven }}$, where $B=1.5 \mathrm{kG}$ is obtained from the energy flux), whereas the number flux in the F13 beam is $10^{20}$ electrons $\mathrm{cm}^{-2} \mathrm{~s}^{-1}$. Even if electrons 
in the coronal loop were globally accelerated, there are only an additional $4 \times$ $10^{19}$ electrons $\mathrm{cm}^{-2} \mathrm{~s}^{-1}$ available. However, this simple assessment does not account for processes that may increase electron density during reconnection and retraction (e.g., Longcope and Guidoni, 2011). Furthermore, high densities between $10^{12}-10^{13} \mathrm{~cm}^{-3}$ have been inferred for some (non-flaring) coronal structures on the Sun (Feldman et al., 1994) and on the flare star EV Lac (Osten et al., 2006). In summary, the energy and number flux requirements for an F13 beam could be met under certain conditions (a dense corona with high magnetic field), but the return-current losses would be significant.

A final issue with the F13 model is the short presence of the hot, blackbodylike emission, which appears only at the end of the impulsive phase between $t \approx 1.8 \mathrm{~s}$ and $t=2.3 \mathrm{~s}$. We expect a coadded burst spectrum to be more directly comparable to spectral observations, which have integration times of $10 \mathrm{~s}$ or more. The coadded spectrum over the $5.1 \mathrm{~s}$ duration of the impulsive heating and the decay phases (dot-dashed light blue line in Figure 1) exhibits a lower color temperature $(7500 \mathrm{~K})$ and larger Balmer jump ratio (2.7) than the instantaneous model spectrum at $t=2.2 \mathrm{~s}$ (Table 1). The coadded F13 spectrum is not as representative of the peak phases of the M-dwarf flares as the instantaneous $t=$ $2.2 \mathrm{~s}$ spectrum, which exhibits prominent $T_{\mathrm{BB}} \approx 10^{4} \mathrm{~K}$ blackbody-like emission as observed. A longer duration of NT electron energy deposition for 5-10 s may produce similar coadded and instantaneous spectra, but the simulation becomes very computationally intensive past $2.3 \mathrm{~s}$ with sustained heating (Section 3.2).

The coadded $5 \mathrm{~s}$ F13 spectrum is more representative of the atmospheric conditions in the gradual decay phase and the very early rise phase. The gradual decay phase of flares exhibits a Balmer jump ratio of $2.6-3.3$ and a blue-optical color temperature of about $8000 \mathrm{~K}$ (K13), which are similar to the observables obtained from the coadded F13 spectrum (Table 1). The coadded F13 spectrum is also consistent with a spectral observation from the very early rise phase of a flare from K13, which we show in Figure 13, K13 characterized two additional properties of flare spectra that can constrain models of the time-evolution: the slope in the NUV at $\lambda<3646 \AA$ and the amount of deviation at red wavelengths (at $\lambda>5000 \AA$ ) from a blackbody fit to the blue-optical range at $\lambda=4000-4800$ $\AA$. Although observations of both the early rise phase and gradual decay phase exhibit a Balmer jump ratio that are larger than during the peak phase, only the early rise exhibits a $T \approx 10000 \mathrm{~K}$ color temperature at $\lambda<3646 \AA$ and a small deviation from the blackbody at redder wavelengths. These two common properties of the average burst model and the early rise phase observation can be seen in Figure 13. However, we expect that flare emission at any particular time (even in the very early rise phase) may consist of a superposition of many individual bursts. In order to conclude that any individual model can explain the time-evolution at any particular time, future modeling work should incorporate a treatment of the spatial evolution of flares, such as the development of two ribbon arcades and sequentially heated white-light kernels. 


\section{Summary and Conclusions}

We have simulated the dynamic and radiative response of an M-dwarf atmosphere subject to a short burst of a constant, high flux $\left(10^{13} \mathrm{erg} \mathrm{cm}^{-2} \mathrm{~s}^{-1}\right)$ of NT electrons with a moderately high low-energy cutoff $(37 \mathrm{keV})$. This simulation produces a heated $(T=12000-13500 \mathrm{~K})$, high density $\left(n_{H}>10^{15} \mathrm{~cm}^{-3}\right)$ "chromospheric condensation" with $N_{H}(n=2)>3 \times 10^{17} \mathrm{~cm}^{-2}, N_{H}(n=3)>10^{17}$ $\mathrm{cm}^{-2}$, and a total physical depth range of $\approx 20 \mathrm{~km}$, resulting in large hydrogen b-f and f-f opacities within a short amount of time $(2.2 \mathrm{~s})$ after the start of non-thermal electron heating. At this time, the "flare photosphere" $\left(\tau_{5000}=1\right)$ is located in the $\mathrm{CC}$ at a height that is nearly $250 \mathrm{~km}$ higher than the pre-flare photosphere, and the density of the CC is over an order of magnitude larger than the material at this height in the pre-flare. Significant heating to $T>10000 \mathrm{~K}$ at high densities (comparable to the density in the very low chromosphere of the pre-flare) is achieved and maintained in the CC and also in the stationary, slightly less dense regions of the atmosphere $\approx 50 \mathrm{~km}$ below the CC (Figures 2 , (4).

The atmospheric response in the F13 model provides a new, accurate description of the radiation field in a flare atmosphere with a dense CC, and moreover, shows that such an atmosphere can produce bright white-light emission with properties that are in good agreement with the observations. The flux spectrum from the F13 model at $t=2.2 \mathrm{~s}$ exhibits a blue-optical color temperature of $T_{\mathrm{BB}} \approx 10^{4} \mathrm{~K}$ between $\lambda=4000-4800 \AA$ and a relatively small Balmer jump ratio ( $\chi_{\text {flare }} \approx 2.0$; Table 1) , both of which are large improvements compared to the range of these parameters in lower beam flux models (F10, F11, and F12 keeping all else the same with the NT electron spectrum). These properties describe typical peak spectra of dMe flares, and we showed the striking match of the instantaneous F13 model at $t=2.2 \mathrm{~s}$ to the observations of one large dMe flare with time-resolved spectra in the impulsive phase (Figure10). The F13 model is the first RHD model to reproduce three generally observed characteristics $\left(T_{\mathrm{BB}}\right.$, $\chi_{\text {flare }}$, and $\mathrm{H} \gamma_{\mathrm{EW} 4170}$; Table 10 self-consistently.

The pioneering gasdvnamic simulations of Livshits et al. (1981) and Katsova, Boiko, and Livshits (1997) produced a CC with similar characteristics to those in the $\mathrm{F} 13 \mathrm{CC}$, and also $\Delta \tau \approx 1$ at $\lambda=4500 \AA$ and $T \approx 9000 \mathrm{~K}$, albeit with $10-30$ times lower NT electron energy fluxes. It is interesting that at least an order of magnitude difference in heating rates gives similar dynamic responses in gasdynamic modeling and radiative-hydrodynamic modeling. Our new radiativehydrodynamic models use a more realistic starting dMe atmosphere with a NLTE treatment of the entire atmosphere (photosphere, chromosphere, transition region, and corona), a detailed treatment of the helium level populations (which are important for regulating the onset of explosive evaporation; see Section 3.2), and a modern parameterization of the NT electron spectrum from RHESSI data. However, a higher cutoff of the NT electron spectrum (as employed in our model) is expected to require a larger flux in order to produce similar dynamics (Fisher, 1989). Finally, the RADYN and RH codes provide a detailed spectrum, which we have shown is important for critically testing the models against the observations, for revealing new atomic physics in flares around the Balmer jump, 
for constraining the charge density in the densest atmospheric regions that are heated during flares (Sections 4.3 4.4), and for understanding how hydrogen recombination appears as a blackbody-like spectrum with $T \approx 10^{4} \mathrm{~K}$ (Section 3.4).

By analyzing the contribution function for the continuum intensity, we conclude that the values of the slope $\left(T_{\mathrm{BB}}\right)$ and Balmer jump ratio $\left(\chi_{\text {flare }}\right)$ of the emergent optical and NUV continuum intensity in the F13 flare atmosphere are due primarily to hydrogen recombination radiation subject to wavelength dependent attenuation from Balmer and Paschen b-f opacities. Laboratory spectra of hydrogen at high density $\left(n \approx 10^{16} \mathrm{~cm}^{-3}\right)$ and temperatures of $T \approx 10^{4}$ K (Wiese, Kelleher, and Paquette, 1972) do not exhibit the blue-optical color temperature of a hot blackbody with $T \approx 10^{4} \mathrm{~K}$. The necessary ingredient to build up optical depth at this density and temperature, and hence produce a $T \approx 10^{4} \mathrm{~K}$ blackbody-like spectrum with a small Balmer jump, is a large physical depth range $(\Delta z)$ of emitting material along the line of sight. Thus, the color temperature in flare spectra does not directly relate to the atmospheric temperature so much as it is an indication of how the amount of emitting material along the line of sight varies as a function of wavelength (indeed, material must be heated to $T \approx 10^{4} \mathrm{~K}$ for hydrogen to be significantly populated out of the ground state into $n=2$ and $n=3$ and generate optical depth). In the CC of the F13 atmosphere, the physical depth range over which continuum emission escapes is $\Delta z_{\mathrm{FWHM}} \approx 1-2 \mathrm{~km}$, which is not present in laboratory experiments. At the blue continuum wavelengths with the lowest opacity (e.g., at $\lambda=4300$ $\AA$ ), the physical depth range over which continuum emission escapes is relatively large in the $\mathrm{CC}$ and extends to lower heights at $z \approx 200 \mathrm{~km}(50 \mathrm{~km}$ below the $\mathrm{CC}$ ). The amount of emitting material along a line of sight can also change due to variations in the density at the heights where continuum emission escapes; in the F13 flare atmosphere, the density is smaller at $z \approx 200 \mathrm{~km}$ compared to the maximum density in the CC (Figure 2). Thus, the integration of the heightdependent properties (as for the contribution function in Figure 4) is important for determining the emergent intensity and flux spectra.

Another important result is that by applying the occupational probability formulae (Hummer and Mihalas, 1988; Dappen. Anderson, and Mihalas, 1987; Tremblay and Bergeron, 2009) to NLTE b-f and b-b Balmer opacities, we can explain the pseudo-continuum properties in the spectral region redward of the Balmer edge $(\lambda>3646 \AA)$ and between the Balmer lines from $\lambda=3750-3900 \AA$. These formulae approximate how wavelengths redward of the Balmer jump experience Balmer b-f opacity in addition to Paschen b-f opacity (and f-f opacity); the atomic processes that allow for this additional Balmer continuum opacity are dissolved upper levels of hydrogen from Stark perturbations by ambient protons and Landau-Zener transitions of electrons between dissolved levels of hydrogen. The amount of Balmer continuum emission redward of the Balmer jump and the amount of high order b-b Balmer emission vary inversely, and therefore the relative amounts of dissolved and undissolved components in spectra provide a new diagnostic of ambient charge density in the white-light emitting, partially ionized regions in the lower flare atmosphere (Section 4.4. Table 22). 
The heating mechanism at high density in dMe flares is still unresolved since the $\mathrm{CC}$ required to produce the observed properties of the white-light emission results from a NT electron beam that would experience a considerable loss of energy from the return-current electric field. The main results of this work are therefore the new interpretation of the blue-optical color temperature and Balmer jump ratio (as the variations in the physical depth range and optical depth as a function of wavelength) and of the apparent pseudo-continuum between the higher order Balmer lines (as Balmer continuum emission due to Landau-Zener transitions of electrons between dissolved levels). Combined with future modeling efforts using a more plausible heating mechanism (Section 7), these insights will allow the spectral observables from individual flares to directly constrain the atmospheric properties in the dense, lower atmosphere.

\section{Future Modeling Improvements}

We plan to develop a grid of short flare bursts using a larger low-energy cutoff of the NT electron spectrum, thereby reducing the number flux of NT electrons (and thus return-current effects) while allowing a larger fraction of energy to penetrate deeper in the atmosphere. A possible alternative is to employ lower NT electron fluxes and explore possible re-acceleration mechanisms in the chromosphere in a similar way as done recently by (Varady et al., 2014). Including additional energy deposition mechanisms, such as NT proton energy deposition (e.g., a neutral beam simulation such as in Karlickv́ et al.,2000) or a combination of energy deposition by other means (e.g., Fletcher and Hudson, 2008) may also be important for producing a similar atmospheric response with a smaller flux of NT electrons. In addition, we plan to incorporate the spatial development of a flare arcade of sequentially heated footpoints (e.g., as in the observations of the Bastille Day solar flare from Qiu et al. (2010) or the cartoon two-ribbon model of the megaflare event in Kowalski et al. (2012)) in order to reproduce the observed timescales of dMe flares, which are longer than the $5 \mathrm{~s}$ burst modeled here.

In future work with the $\mathrm{RH}$ code, we intend to improve the implementation of occupational probability theory and Stark broadening. Hubeny, Hummer, and Lanz (1994) fully extended the occupational probability formalism to NLTE, and we suggest that flare modeling codes developed in the future should similarly include occupational probability formalism in the non-equilibrium rate equation (following Equation 2.23 in Hubeny, Hummer, and Lanz, 1994) in addition to the NLTE b-b and b-f opacity and emissivity. Here, our goal was to demonstrate that large improvements in the NUV model spectrum result from implementing the simple modifications to the opacity and emissivity given in Dappen, Anderson, and Mihalas (1987) and Tremblay and Bergeron (2009). We will also follow the work of Tremblay and Bergeron (2009) and Seaton (1990) and renormalize the Stark profiles of Vidal. Cooper, and Smith (1973) using $\beta_{\text {crit }}$ as an integration limit, which leads to significantly narrower higher order Balmer lines ( $c f$. Figure 5 of Tremblay and Bergeron, 2009) The theory of Vidal. Cooper, and Smith (1973) is only valid to first order when the line wings do not overlap, since the LandauZener continuum accounts for larger perturbations. Therefore, too much emission 
is probably produced between the Balmer lines between $\lambda=3800-4000 \AA$ in the model spectrum in Figure 11. Between $\lambda=3646-3800 \AA$, the low values of $w_{n}$ (that multiply into the b-b opacity) lead to only a small amount of blended Balmer line wings compared to the Landau-Zener continuum, so the error is not important. Finally, the linear Stark broadening theory of the Balmer lines using the analytic approximations of Sutton (1978) as Voigt damping parameters should be replaced by the full Stark profiles from Vidal. Cooper, and Smith (1973) and Lemke (1997) for the conditions present in flare atmospheres, in order to give accurate diagnostics on the electron density for unblended Balmer lines.

In future work with the RADYN code (Allred et al. 2015, in preparation), we intend to include the following improvements to the modeled physics: the cooling from $\mathrm{Mg}$ II and Fe II ions, an improved optically thin radiative loss function, nonthermal ionization of He I and He II, return-current heating, magnetic mirroring of the NT electrons, and the fully relativistic Fokker-Planck solution to energy deposition and pitch angle scattering of NT electrons. 
Table 1. Observables calculated from the model flux spectra. These values have been obtained without subtracting the preflare spectrum.

\begin{tabular}{llll}
\hline Model (time step; phase) & $\chi_{\text {flare }}$ & $T_{\mathrm{BB}}(\mathrm{K})$ & $\mathrm{H} \gamma_{\mathrm{EW} 170}$ \\
\hline F11 $(t=2.2 \mathrm{~s}$; impulsive peak) & 9.2 & 5300 & 150 \\
F12 $(t=2.2 \mathrm{~s}$; impulsive peak) & 7.4 & 5700 & 85 \\
F13 $(t=0.2 \mathrm{~s}$; impulsive early, rise 1$)$ & 6.0 & 6300 & 70 \\
F13 $(t=1.2 \mathrm{~s}$; impulsive late, rise 2$)$ & 3.3 & 7400 & 40 \\
F13 $(t=2.2 \mathrm{~s}$; impulsive peak $)$ & 2.0 & 9300 & 20 \\
F13 $(t=4 \mathrm{~s}$; gradual decay) & 3.3 & 5400 & 60 \\
F13 (average burst) & 2.7 & 7500 & 30
\end{tabular}

Table 2. The Landau-Zener Balmer decrement $(\mathrm{H} 11 / \mathrm{H} \gamma)$ in the optically thick, isothermal, LTE limit. Here, we give only the charged component of $w_{n}$ (Equation (4), averaged for the range of temperatures), ignoring the minor dependence on the destruction of levels from collisions with other neutrals.

\begin{tabular}{llllll}
\hline$n_{p}\left(\mathrm{~cm}^{-3}\right)$ & $w_{n=11}$ & $T=10000 \mathrm{~K}$ & $T=12500 \mathrm{~K}$ & $T=15000 \mathrm{~K}$ & $T=20000 \mathrm{~K}$ \\
\hline $10^{13}$ & 1.00 & 0.30 & 0.33 & 0.35 & 0.37 \\
$10^{14}$ & 0.98 & 0.30 & 0.32 & 0.34 & 0.37 \\
$5 \times 10^{14}$ & 0.85 & 0.26 & 0.28 & 0.30 & 0.32 \\
$10^{15}$ & 0.69 & 0.21 & 0.23 & 0.24 & 0.25 \\
$2.5 \times 10^{15}$ & 0.35 & 0.11 & 0.12 & 0.12 & 0.12 \\
$5 \times 10^{15}$ & 0.15 & 0.05 & 0.05 & 0.05 & 0.05
\end{tabular}




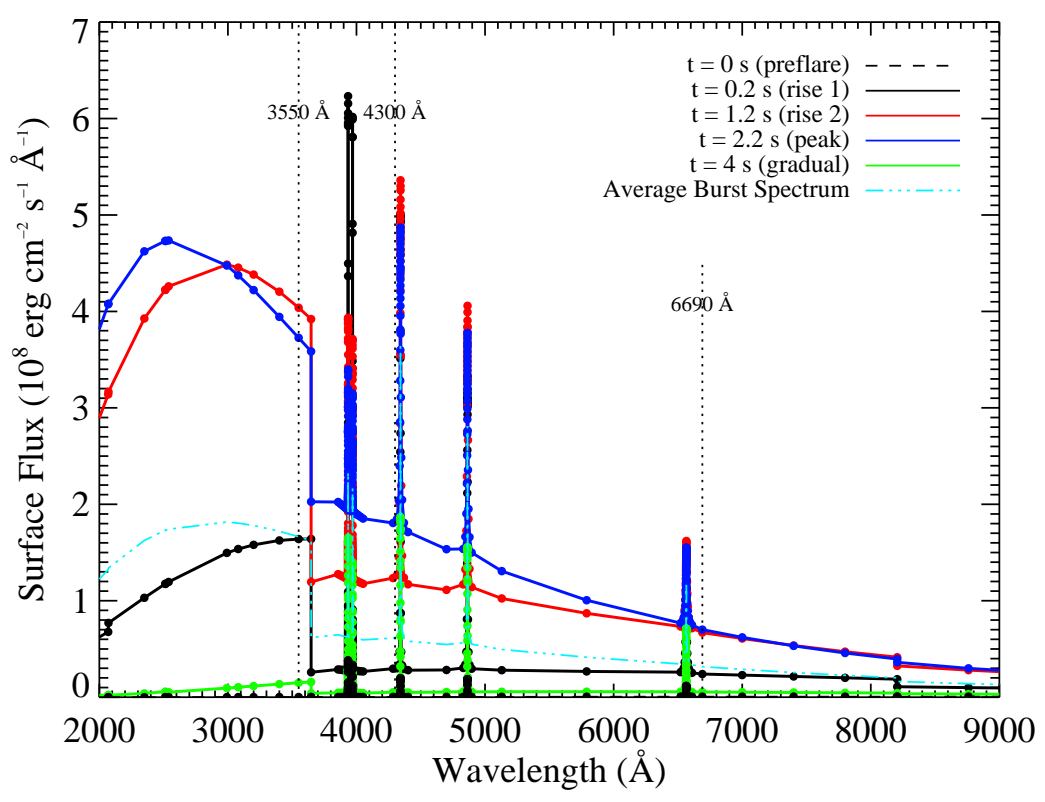

Figure 1. The evolution of the NUV and optical flux spectra during the F13 simulation. The observable parameters extracted from these spectra are given in Table 1 The coadded (average) burst spectrum is discussed in Section 5 The preflare spectrum is not visible on this scale. The vertical dotted lines indicate the $\operatorname{NUV}(\lambda=3550 \AA)$, blue-optical $(\lambda=4300 \AA)$, and red-optical $(\lambda=6690 \AA)$ wavelengths used in the continuum analysis (Section 3.3 ). 

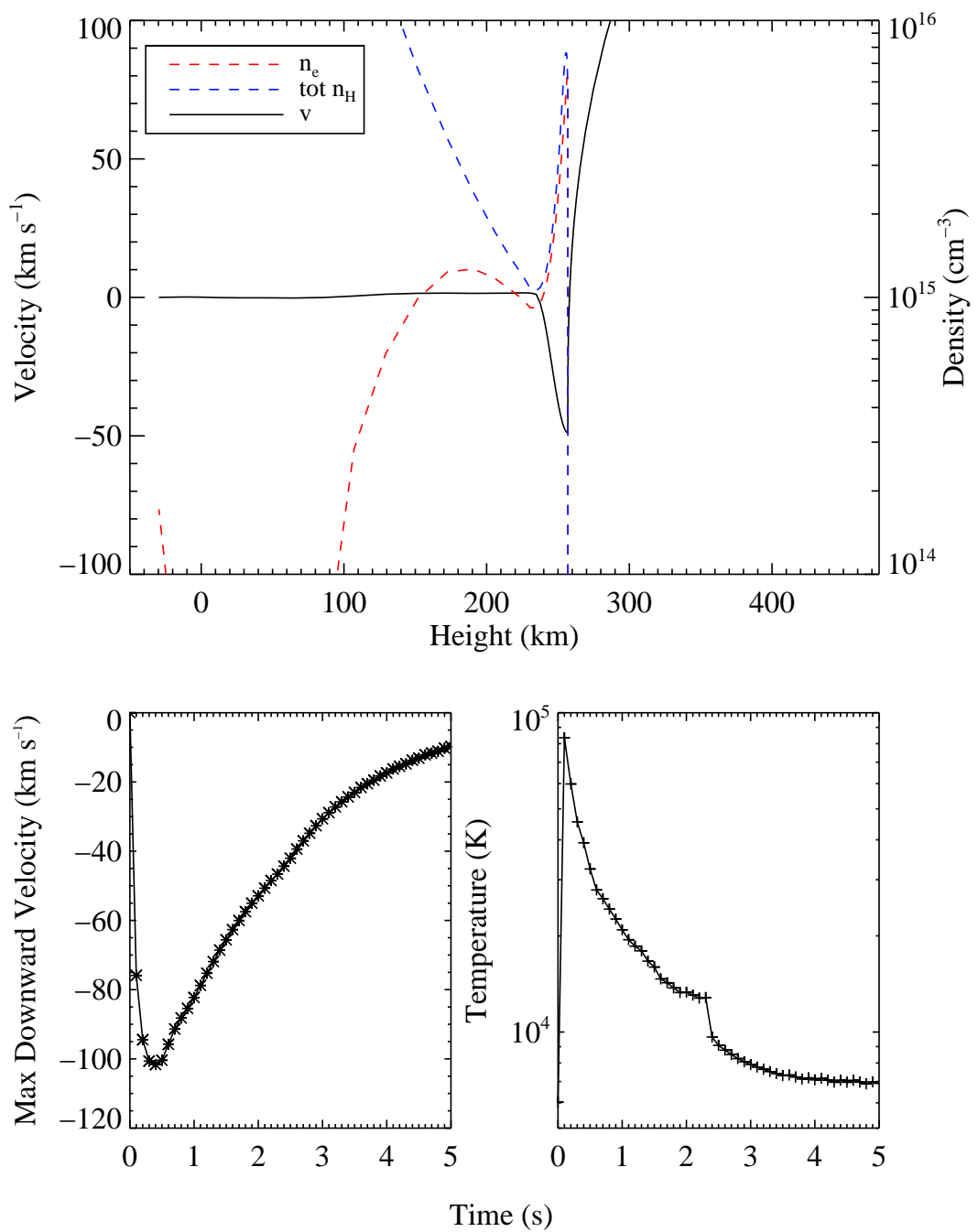

Figure 2. Top: The velocity field in the lower atmosphere at $t=2.2 \mathrm{~s}$ shows a chromospheric condensation (CC) with a large hydrogen and electron density exceeding $5 \times 10^{15} \mathrm{~cm}^{-3}$. Bottom left: The time-dependence of the velocity in the highest density zone in the CC. Bottom right: The time-dependence of the temperature in the highest density zone in the CC. The velocity of the CC changes smoothly through the burst while the temperature reflects the cutoff of the NT electron energy deposition at $t=2.3 \mathrm{~s}$. 


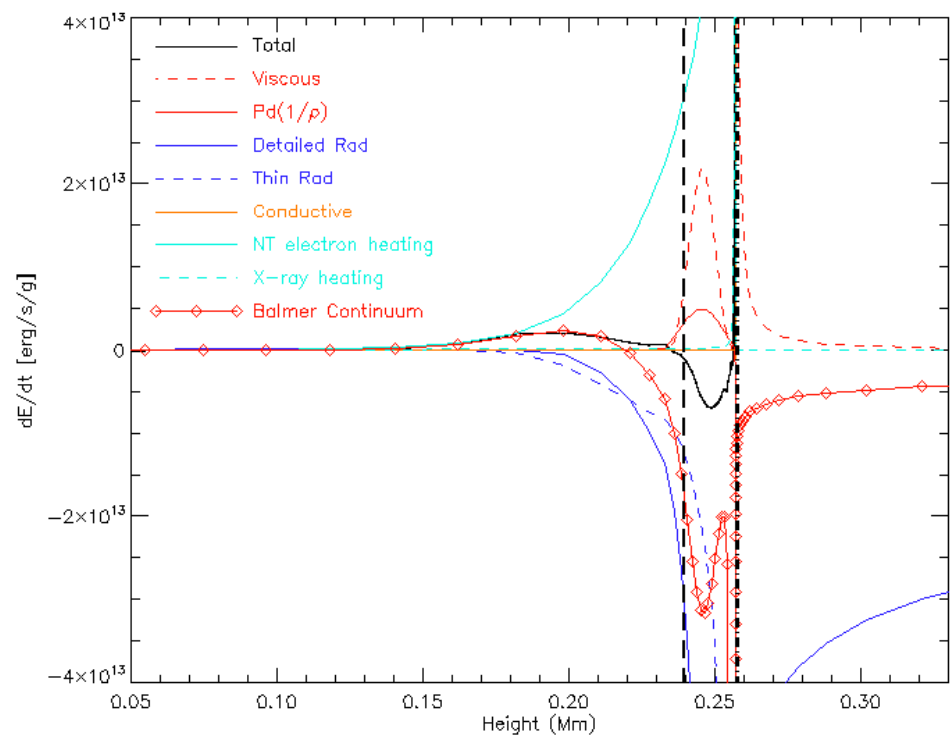

Figure 3. Contributions to the energy balance for $t=2.2 \mathrm{~s}$ in the F13 simulation. These curves show the net amount for each component. For the detailed radiation, the heating and cooling are integrated over the wavelengths of the transitions. The Balmer continuum (red diamonds) heating/cooling is shown as a separate curve from the total detailed radiation curve (which includes the Balmer continuum). The CC, with velocities lower than $5 \mathrm{~km} \mathrm{~s}^{-1}$, is material between $z=239.4 \mathrm{~km}$ (long dashed vertical black line) and $257.4 \mathrm{~km}$ (thick vertical dash-dotted line). 


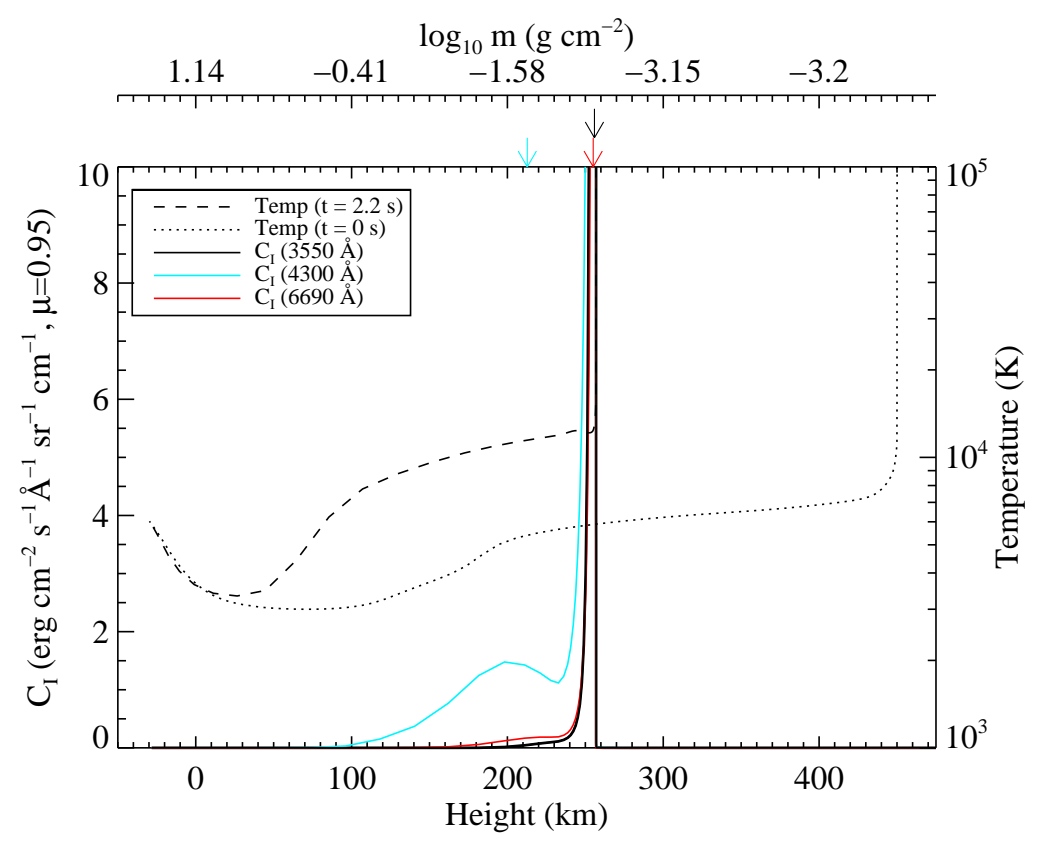

Figure 4. The contribution to the emergent intensity for three continuum wavelengths in the NUV, blue, and red at $t=2.2 \mathrm{~s}$ compared to the temperature structure (for the same height range as in Figure 21). The column mass scale for $t=2.2 \mathrm{~s}$ is shown at the top axis. The arrows indicate where $\tau=1$ occurs for each wavelength. The contribution functions in the CC $(z \approx 239-257 \mathrm{~km})$ are extremely thin and extend off the top of the plot (see text). 


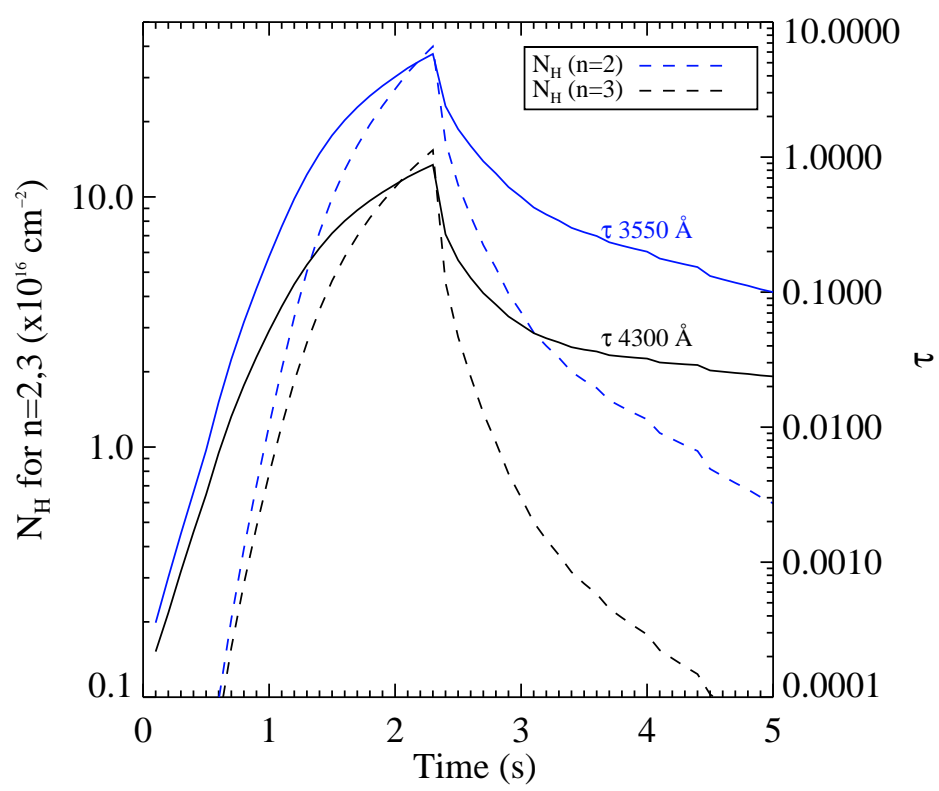

Figure 5. The evolution of column density for $n=2$ and $n=3$ of hydrogen (left axis, dashed lines) and optical depth (right axis, solid lines) for atmospheric heights in the CC with velocities $<-5 \mathrm{~km} \mathrm{~s}^{-1}$. At $t=2.2 \mathrm{~s}$, the CC has an extent of $18 \mathrm{~km}$ from $239 \mathrm{~km}$ to 257 $\mathrm{km}$ and has temperatures $>12000 \mathrm{~K}$. Approximately $3.5 \times 10^{12} \mathrm{erg} \mathrm{cm}^{-2} \mathrm{~s}^{-1}$ of NT electron energy flux is being deposited within the $\mathrm{CC}$ at this time. 

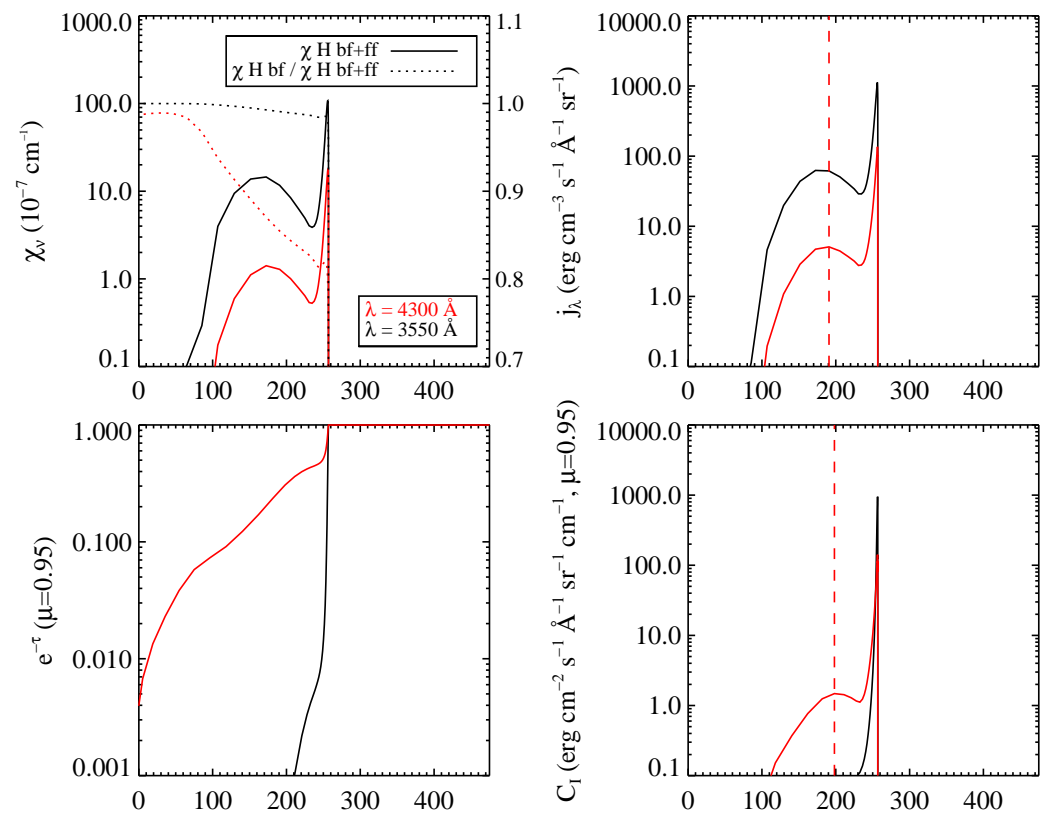

Height $(\mathrm{km})$

Figure 6. The contribution function (bottom right) for $4300 \AA$ and $3550 \AA$ is broken up into three parts: opacity (top left), emissivity (top right), and the attenuation (bottom left). The ratios of hydrogen b-f opacity to total hydrogen (b-f and f-f) opacity are shown in the top left panel (right axis). In the stationary flare layers $(z \approx 190 \mathrm{~km})$, there is a large emissivity at $\lambda=3550 \AA$ but also a very large attenuation due to photoionizations of hydrogen from $n=2$ in the higher layers of the CC. The peaks of the emissivity and contribution function in the stationary layers for $\lambda=4300 \AA$ are indicated by vertical dashed lines (see text). 

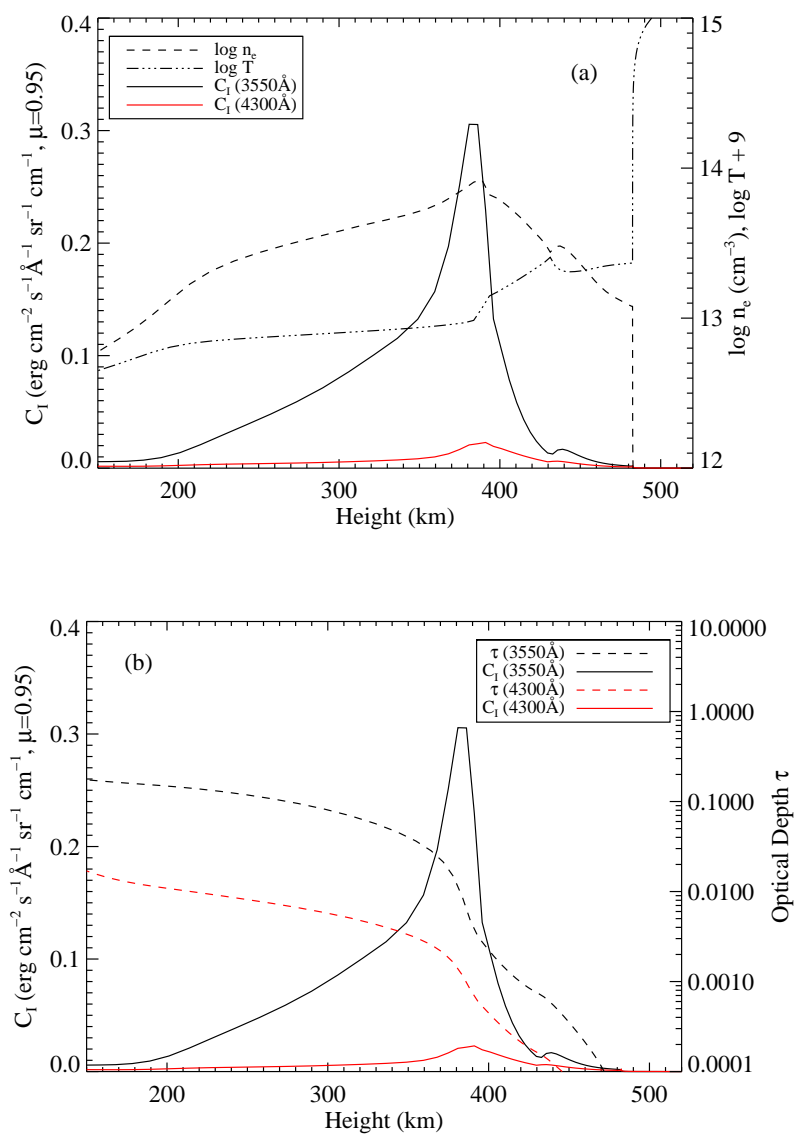

Figure 7. The contribution function for the F11 model at $\mu=0.95$ and $t=2.2 \mathrm{~s}$ compared to the (a) electron density and temperature and (b) optical depth. Only heights corresponding to the flare chromosphere, transition region, and lower corona are shown. 


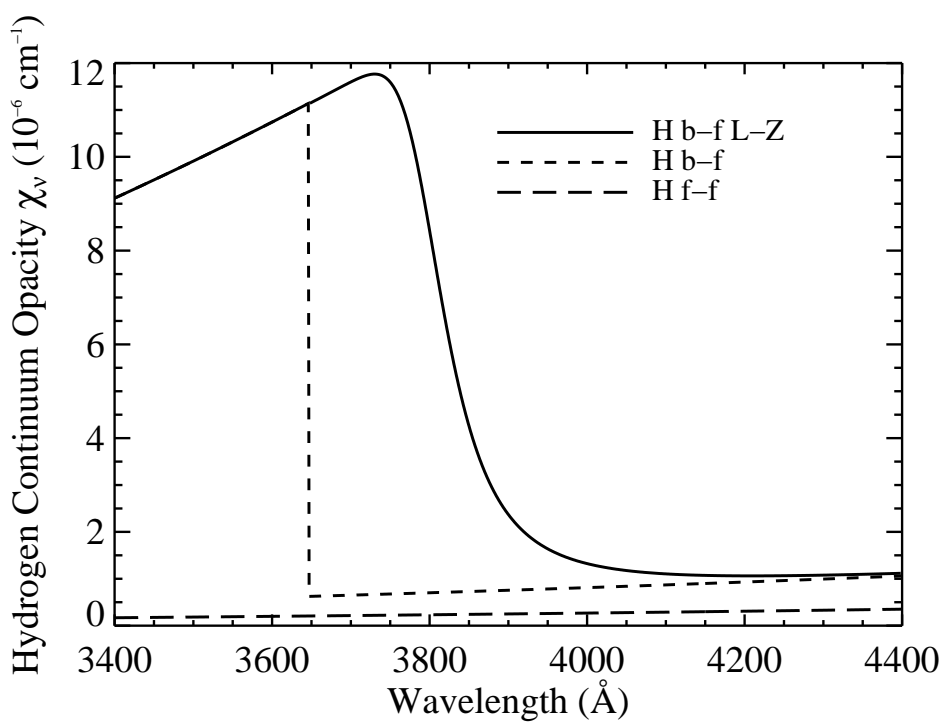

Figure 8. The hydrogen b-f continuum opacity accounting for Landau-Zener (L-Z) transitions, at $t=2.2 \mathrm{~s}$ in the F13 simulation, at a height of $255.4 \mathrm{~km}\left(\log _{10} m=-2.57 \mathrm{~g} \mathrm{~cm}^{-2}\right)$ which is the maximum of $C_{I}(\lambda=3550) \Delta z \times C_{I}(\lambda=4300) \Delta z$ where $\Delta z$ is the vertical extent of a grid cell. The values of $n_{p}$ and $T_{e}$ are respectively, $5.6 \times 10^{15} \mathrm{~cm}^{-3}$ and $12,770 \mathrm{~K}$. At this height, hydrogen is nearly $75 \%$ ionized. Also shown are the hydrogen f-f opacity and the hydrogen b-f opacity without accounting for opacity effects from Landau-Zener transitions at this height. At this layer, $\tau_{3550}=1.1$ and $\tau_{4300}=0.2$. 


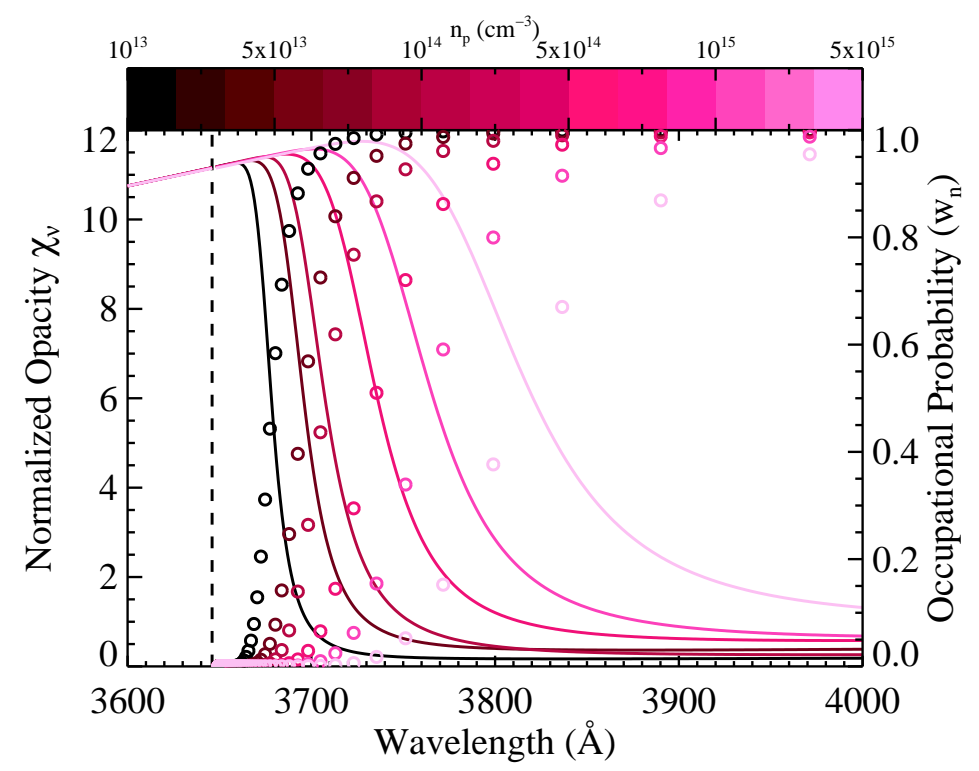

Figure 9. The hydrogen b-f opacity accounting for Landau-Zener transitions at representative times and heights in the F11 and F13 simulations. Each curve corresponds to approximately the ambient charge density at each of the tick marks in the colorbar. The opacity curves have been scaled to a common value at $\lambda=3600 \AA$ to illustrate the wavelength shift of the opacity maximum compared to the Balmer b-f edge (vertical dashed line) without accounting for Landau-Zener transitions. The occupational probabilities (right axis) of the upper levels of the Balmer transitions are shown as circles (for reference H10 is at $\lambda \approx 3800 \AA$ ). 


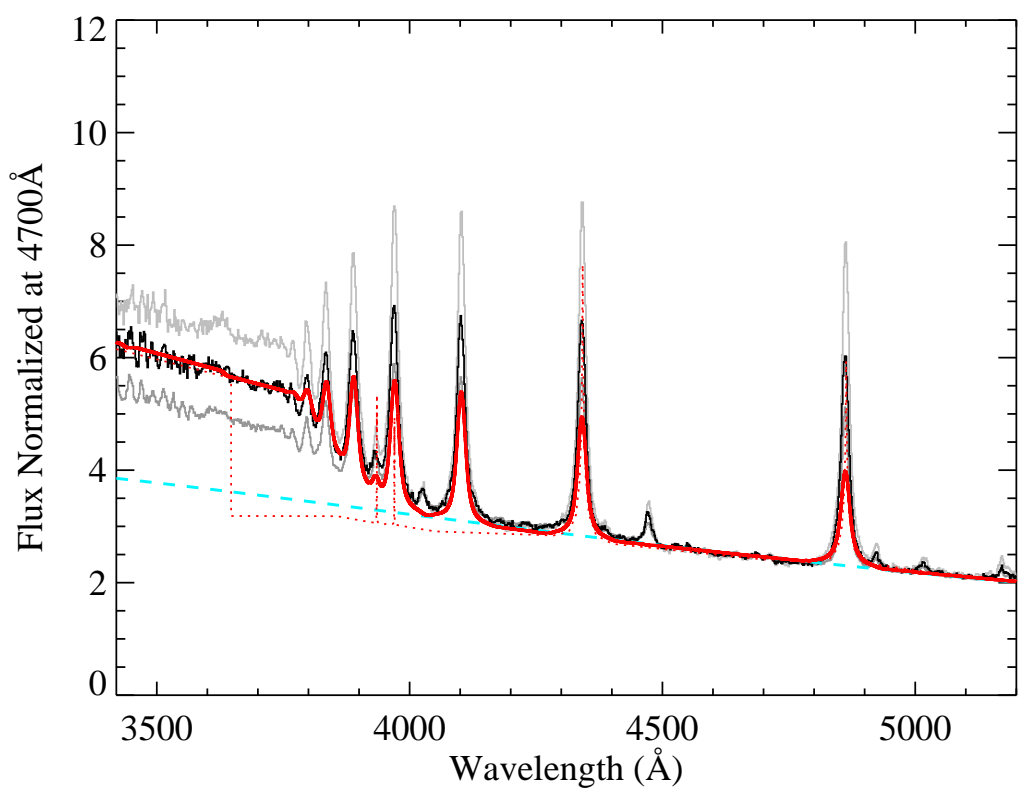

Figure 10. RH calculation with a 20-level hydrogen atom and the b-f and b-b opacities including Landau-Zener transitions, during a snapshot of the F13 simulation at $t=2.2 \mathrm{~s}$ (solid red). A $T=10400 \mathrm{~K}$ blackbody fit to continuum windows at $\lambda=4000-4800 \AA$ is also shown (dashed light blue), along with the RADYN prediction with a 6-level hydrogen atom (red dotted line). Instrumental broadening has been applied to the red spectrum. Several observations during the rise and peak phases of the IF3 flare of K13 are shown: peak spectrum is $\mathrm{S} \# 31$ from K13 (dark gray), mid rise phase is $\mathrm{S} \# 27$ from K13 (light gray), and late rise phase is S\#28 from K13 (black). All spectra are scaled to the flux at $\lambda=4690-4710 \AA$. 


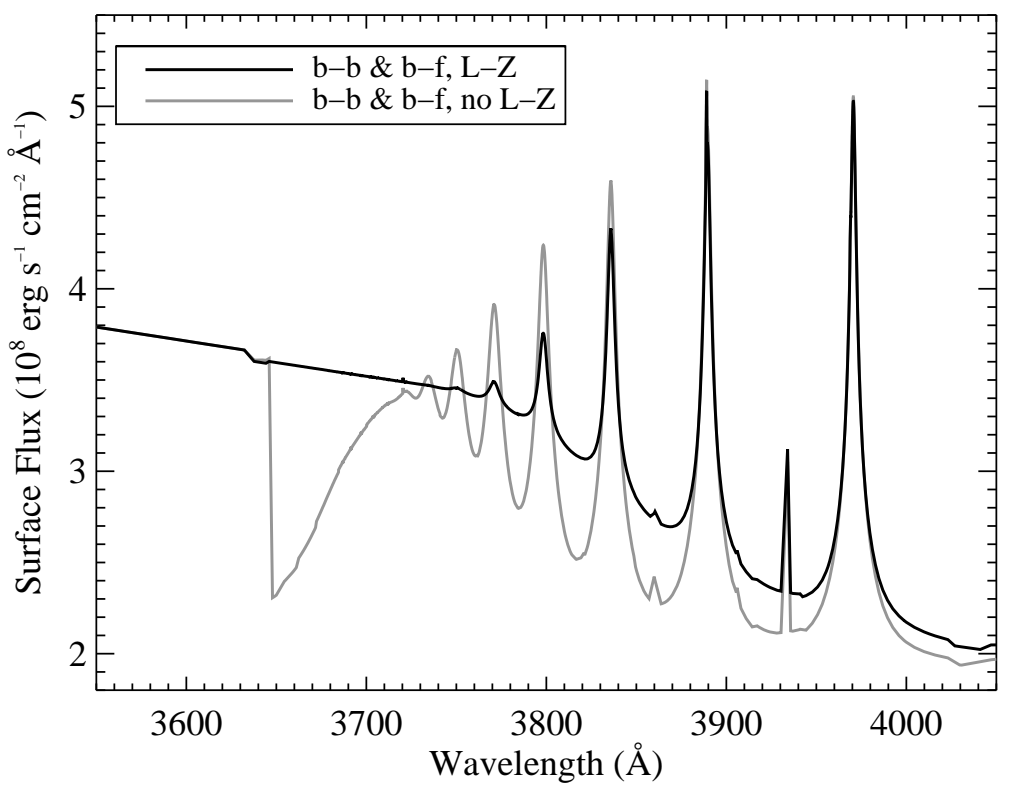

Figure 11. RH spectra of the Balmer jump region without instrumental convolution applied and with a magnified flux scale. Black line: Electric field effects on b-b opacities and Landau-Zener transition effects on b-b and b-f opacities from critical electric fields. H11 is the bluest identifiable Balmer line. Gray line: Electric field effects on b-b opacities and Landau-Zener transitions not included. H13 is the bluest identifiable Balmer line. Including Landau-Zener transitions in the b-b and b-f opacities predicts more continuum emission between $3646 \AA$ and $3720 \AA$, whereas not accounting for Landau-Zener transitions produces a "blue continuum bump" at $\lambda \approx 3720 \AA$ from the blending of Stark-broadened Balmer lines. Instrumental broadening is not shown; it broadens the Balmer lines but does not have a strong effect on the amount of continuum between the lines. 


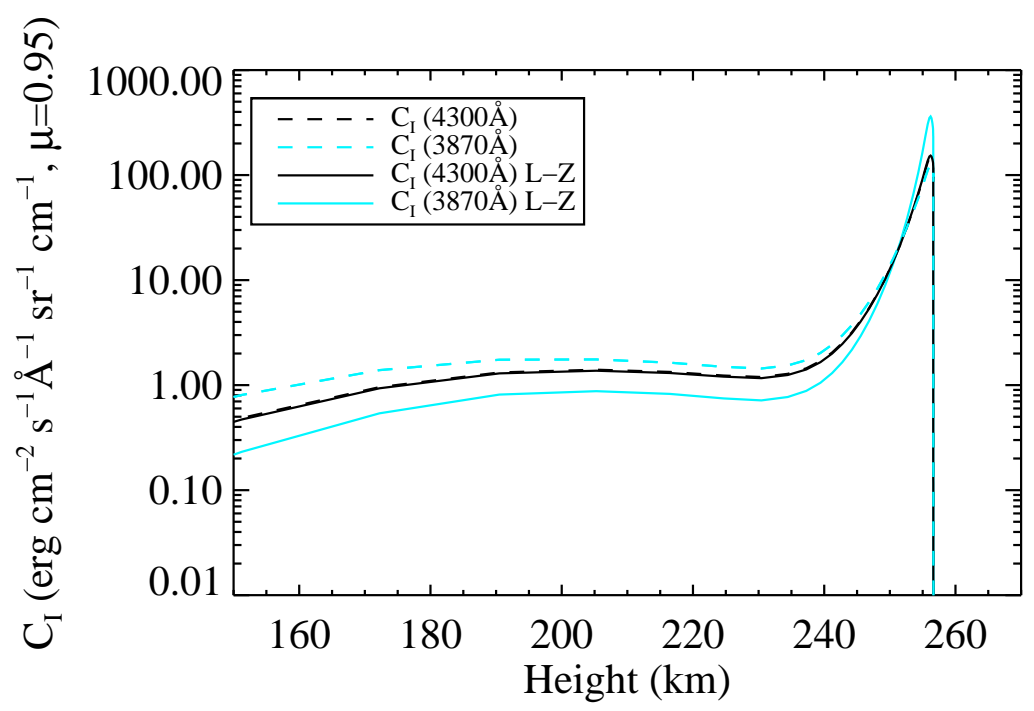

Figure 12. The contribution function at $\lambda=3870 \AA$ and $4300 \AA$ accounting for opacity effects from Landau-Zener transitions (solid) compared to the original calculation (dashed). With L-Z transitions, the additional Balmer opacity at $\lambda=3870 \AA$ causes more emission to escape from the CC and less from the stationary flare layers than without the L-Z transitions. The contribution function at $\lambda=4300 \AA$ is not affected. 


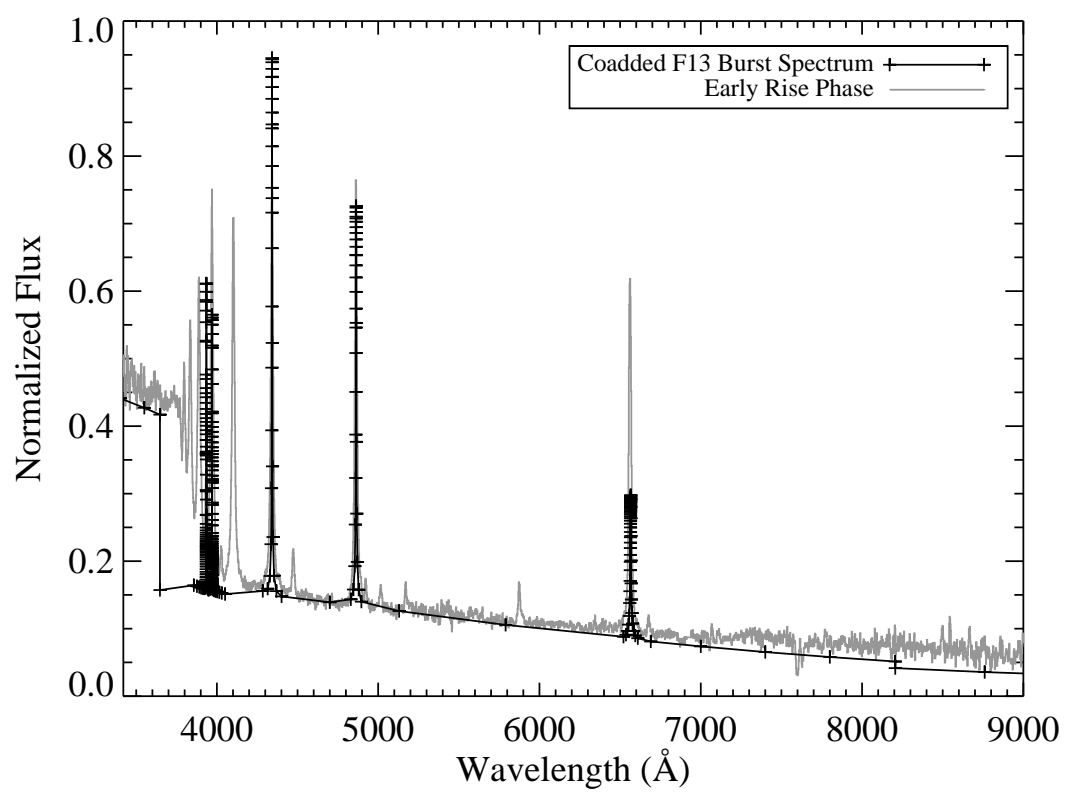

Figure 13. The coadded (average) model F13 spectra (from RADYN; instrumental broadening is not applied) over $5 \mathrm{~s}$ compared to the early rise spectrum (S\#26) during the IF3 flare from K13. The coadded model spectrum has a larger Balmer jump and lower optical color temperature than the instantaneous model spectrum at $t=2.2 \mathrm{~s}$ in Figure 10 The flux at $\lambda<3646 \AA$ increases towards bluer wavelengths in the model and observation. Note that the opacity effects from Landau-Zener transitions are not included here because statistical equilibrium is a poor approximation during the early time steps in the model (Section 4.3).

Acknowledgements AFK thanks the Science Organizing Committee of the Solar and Stellar Flares meeting in Prague, Czech Republic for the opportunity to present this work. We thank an anonymous referee for clarifications and comments that helped improve this work. AFK thanks Dr. Petr Heinzel and Dr. Hans Ludwig for bringing hot star modeling papers of Stark broadening to his attention. We thank Drs. Adrian Daw, Eric Agol, Ellen Zweibel, and Jeremiah Murphy for helpful discussions and Dr. William Abbett for several IDL routines used in the analysis. AFK also acknowledges helpful discussions at the International Space Science Institute with Dr. Lyndsay Fletcher's Solar and Stellar Flares team and with Dr. Sven Wedemeyer-Bohm's Magnetic Activity of M-type Dwarf Stars and the Influence on Habitable Extra-solar Planets team. This research was supported by an appointment to the NASA Postdoctoral Program at the Goddard Space Flight Center, administered by Oak Ridge Associated Universities through a contract with NASA, and by the University of Maryland Goddard Planetary Heliophysics Institute (GPHI) Task 132. 


\section{References}

Abbett, W.P., Hawley, S.L.: 1999, Dynamic Models of Optical Emission in Impulsive Solar Flares. Astrophys. J. 521, 906. DOI ADS.

Aller, L.H.: 1963, Astrophysics. The atmospheres of the sun and stars, 2nd edn. Ronald Press, New York. ADS

Allred, J.C., Hawley, S.L., Abbett, W.P., Carlsson, M.: 2005, Radiative Hydrodynamic Models of the Optical and Ultraviolet Emission from Solar Flares. Astrophys. J. 630, 573. DOI ADS

Allred, J.C., Hawley, S.L., Abbett, W.P., Carlsson, M.: 2006, Radiative Hydrodynamic Models of Optical and Ultraviolet Emission from M Dwarf Flares. Astrophys. J. 644, 484. DOI ADS

Aschwanden, M.J., Kliem, B., Schwarz, U., Kurths, J., Dennis, B.R., Schwartz, R.A.: 1998, Wavelet Analysis of Solar Flare Hard X-Rays. Astrophys. J. 505, 941. DOI. ADS

Avrett, E.H., Machado, M.E., Kurucz, R.L.: 1986, Chromospheric flare models. In: Neidig, D.F. (ed.) The lower atmosphere of solar flares, p. 216 - 281, 216. ADS

Bengtson, R.D., Tannich, J.D., Kepple, P.: 1970, Comparison Between Measured and Theoretical Stark-Broadened Profiles of $\mathrm{H}_{6}-\mathrm{H}_{12}$ Emitted from a Low-Density Plasma. Phys. Rev. A 1, 532. DOI ADS

Brown, J.C.: 1971, The Deduction of Energy Spectra of Non-Thermal Electrons in Flares from the Observed Dynamic Spectra of Hard X-Ray Bursts. Solar Phys. 18, 489. DOI ADS

Carlsson, M.: 1998, Radiative Transfer and Radiation Hydrodynamics. In: Vial, J.C., Bocchialini, K., Boumier, P. (eds.) Space Solar Physics: Theoretical and Observational Issues in the Context of the SOHO Mission, Lecture Notes in Physics, Berlin Springer Verlag 507, 163. DOI ADS

Carlsson, M., Rutten, R.J.: 1992, Solar hydrogen lines in the infrared. Astron. Astrophys. 259, L53. ADS

Carlsson, M., Stein, R.F.: 1992, Non-LTE radiating acoustic shocks and CA II K2V bright points. Astrophys. J. Lett. 397, L59. DOI ADS

Carlsson, M., Stein, R.F.: 1994, Radiation shock dynamics in the solar chromosphere - results of numerical simulations. In: Carlsson, M. (ed.) Chromospheric Dynamics, University, Oslo, 47. ADS

Carlsson, M., Stein, R.F.: 1995, Does a nonmagnetic solar chromosphere exist? Astrophys. J. Lett. 440, L29. DOI ADS.

Carlsson, M., Stein, R.F.: 1997, Formation of Solar Calcium H and K Bright Grains. Astrophys. J. 481, 500. DOI ADS

Carlsson, M., Stein, R.F.: 2002, Dynamic Hydrogen Ionization. Astrophys. J. 572, 626. DOI ADS

Christian, D.J., Mathioudakis, M., Jevremović, D., Dupuis, J., Vennes, S., Kawka, A.: 2003, The Extreme-Ultraviolet Continuum of a Strong Stellar Flare. Astrophys. J. Lett. 593, L105. DOI ADS

Cram, L.E., Woods, D.T.: 1982, Models for stellar flares. Astrophys. J. 257, 269. DOI ADS.

Dalgarno, A., Griffing, G.W.: 1958, Energy per Ion Pair for Electron and Proton Beams in Atomic Hydrogen. Roy. Soc. London Proc. Ser. A 248, 415. DOI ADS.

Dappen, W., Anderson, L., Mihalas, D.: 1987, Statistical mechanics of partially ionized stellar plasma - The Planck-Larkin partition function, polarization shifts, and simulations of optical spectra. Astrophys. J. 319, 195. DOI ADS

Dennis, B.R., Zarro, D.M.: 1993, The Neupert effect - What can it tell us about the impulsive and gradual phases of solar flares? Solar Phys. 146, 177. DOI ADS

Dere, K.P., Landi, E., Mason, H.E., Monsignori Fossi, B.C., Young, P.R.: 1997, CHIANTI an atomic database for emission lines. Astron. Astrophys. Suppl. 125, 149. DOI ADS

Donati-Falchi, A., Falciani, R., Smaldone, L.A.: 1985, Analysis of the optical spectra of solar flares. IV - The 'blue' continuum of white light flares. Astron. Astrophys. 152, 165. ADS

Donati-Falchi, A., Smaldone, L.A., Falciani, R.: 1984, Analysis of the optical spectra of solar flares. II - The energetics of the June 4, 1980 white light flare. Astron. Astrophys. 131, 256. ADS

Dorfi, E.A., Drury, L.O.: 1987, Simple adaptive grids for 1-D initial value problems. Journal of Computational Physics 69, 175. DOI ADS.

Doyle, J.G., Butler, C.J., Bryne, P.B., van den Oord, G.H.J.: 1988, Rotational modulation and flares on RS CVn and BY DRA systems. Astron. Astrophys. 193, 229. ADS 
Drake, S.A., Ulrich, R.K.: 1980, The emission-line spectrum from a slab of hydrogen at moderate to high densities. Astrophys. J. Supp. Series 42, 351. DOI ADS

Eason, E.L.E., Giampapa, M.S., Radick, R.R., Worden, S.P., Hege, E.K.: 1992, Spectroscopic and photometric observations of a five-magnitude flare event on UV Ceti. Astron. J. 104, 1161. DOI ADS.

Emslie, A.G.: 1978, The collisional interaction of a beam of charged particles with a hydrogen target of arbitrary ionization level. Astrophys. J. 224, 241. DOI. ADS.

Fang, C., Hénoux, J.C., Gan, W.Q.: 1993, Diagnostics of non-thermal processes in chromospheric flares. 1. Hoe and Call K line profiles of an atmosphere bombarded by $10-500 \mathrm{keV}$ electrons. Astron. Astrophys. 274, 917. ADS

Feldman, U., Hiei, E., Phillips, K.J.H., Brown, C.M., Lang, J.: 1994, Very impulsive solar flares observed with the YOHKOH spacecraft. Astrophys. J. 421, 843. DOI. ADS.

Fisher, G.H.: 1989, Dynamics of flare-driven chromospheric condensations. Astrophys. J. 346, 1019. DOI ADS.

Fisher, G.H., Canfield, R.C., McClymont, A.N.: 1985, Flare Loop Radiative Hydrodynamics Part Six - Chromospheric Evaporation due to Heating by Nonthermal Electrons. Astrophys. J. 289, 425. DOI ADS

Fletcher, L., Hudson, H.S.: 2008, Impulsive Phase Flare Energy Transport by Large-Scale Alfvén Waves and the Electron Acceleration Problem. Astrophys. J. 675, 1645. DOI. ADS

Fuhrmeister, B., Liefke, C., Schmitt, J.H.M.M., Reiners, A.: 2008, Multiwavelength observations of a giant flare on CN Leonis. I. The chromosphere as seen in the optical spectra. Astron. Astrophys. 487, 293. DOI. ADS

Fuhrmeister, B., Lalitha, S., Poppenhaeger, K., Rudolf, N., Liefke, C., Reiners, A., Schmitt, J.H.M.M., Ness, J.-U.: 2011, Multi-wavelength observations of Proxima Centauri. Astron. Astrophys. 534, A133. DOI. ADS.

Gan, W.Q., Fang, C.: 1990, A hydrodynamic model of the gradual phase of the solar flare loop. Astrophys. J. 358, 328. DOI ADS.

Gan, W.Q., Rieger, E., Zhang, H.Q., Fang, C.: 1992, The role of chromospheric condensations in the continuum emission of white-light flares. Astrophys. J. 397, 694. DOI. ADS.

García-Alvarez, D., Jevremović, D., Doyle, J.G., Butler, C.J.: 2002, Observations and modelling of a large optical flare on AT Microscopii. Astron. Astrophys. 383, 548. DOI. ADS.

Gritsyk, P.A., Somov, B.V.: 2014, Reverse-current effect in present-day models of solar flares: Theory and high-accuracy observations. Astronomy Letters 40, 499. DOI ADS

Guedel, M., Benz, A.O., Schmitt, J.H.M.M., Skinner, S.L.: 1996, The Neupert Effect in Active Stellar Coronae: Chromospheric Evaporation and Coronal Heating in the dMe Flare Star Binary UV Ceti. Astrophys. J. 471, 1002. DOI ADS

Gustafsson, B.: 1973, A FORTRAN Program for Calculating "Continuous" Absorption Coefficients of Stellar Atmospheres. Uppsala Astr. Obs. Ann. 5.

Hawley, S.L., Fisher, G.H.: 1992, X-ray-heated models of stellar flare atmospheres - Theory and comparison with observations. Astrophys. J. Supp. Series 78, 565. DOI ADS.

Hawley, S.L., Fisher, G.H.: 1994, Solar flare model atmospheres. Astrophys. J. 426, 387. DOI ADS

Hawley, S.L., Pettersen, B.R.: 1991, The great flare of 1985 April 12 on AD Leonis. Astrophys. J. 378, 725. DOI ADS

Hawley, S.L., Fisher, G.H., Simon, T., Cully, S.L., Deustua, S.E., Jablonski, M., Johns-Krull, C.M., Pettersen, B.R., Smith, V., Spiesman, W.J., Valenti, J.: 1995, Simultaneous ExtremeUltraviolet Explorer and Optical Observations of AD Leonis: Evidence for Large Coronal Loops and the Neupert Effect in Stellar Flares. Astrophys. J. 453, 464. DOI ADS

Hawley, S.L., Allred, J.C., Johns-Krull, C.M., Fisher, G.H., Abbett, W.P., Alekseev, I., Avgoloupis, S.I., Deustua, S.E., Gunn, A., Seiradakis, J.H., Sirk, M.M., Valenti, J.A.: 2003, Multiwavelength Observations of Flares on AD Leonis. Astrophys. J. 597, 535. DOI ADS.

Hawley, S.L., Davenport, J.R.A., Kowalski, A.F., Wisniewski, J.P., Hebb, L., Deitrick, R., Hilton, E.J.: 2014, Kepler Flares. I. Active and Inactive M Dwarfs. Astrophys. J. 797, 121. DOI ADS

Heinzel, P., Kleint, L.: 2014, Hydrogen Balmer Continuum in Solar Flares Detected by the Interface Region Imaging Spectrograph (IRIS). Astrophys. J. Lett. 794, L23. DOI ADS

Holman, G.D.: 2012, Understanding the Impact of Return-current Losses on the X-Ray Emission from Solar Flares. Astrophys. J. 745, 52. DOI. ADS

Holman, G.D., Sui, L., Schwartz, R.A., Emslie, A.G.: 2003, Electron Bremsstrahlung Hard X-Ray Spectra, Electron Distributions, and Energetics in the 2002 July 23 Solar Flare. Astrophys. J. Lett. 595, L97. DOI ADS 
Houdebine, E.R.: 1992, Investigating the spectroscopic signatures of stellar flares. Irish Astron. J. 20, 213. ADS

Hubeny, I., Hummer, D.G., Lanz, T.: 1994, NLTE model stellar atmospheres with line blanketing near the series limits. Astron. Astrophys. 282, 151. ADS.

Hummer, D.G., Mihalas, D.: 1988, The equation of state for stellar envelopes. I - an occupation probability formalism for the truncation of internal partition functions. Astrophys. J. 331, 794. DOI ADS

Ireland, J., Tolbert, A.K., Schwartz, R.A., Holman, G.D., Dennis, B.R.: 2013, Estimating the Properties of Hard X-Ray Solar Flares by Constraining Model Parameters. Astrophys. J. 769, 89. DOI ADS

Jevremovic, D., Butler, C.J., Drake, S.A., O'Donoghue, D., van Wyk, F.: 1998, Ultraviolet and optical flares on GL 866. Astron. Astrophys. 338, 1057. ADS

Johns-Krull, C.M., Valenti, J.A.: 1996, Detection of Strong Magnetic Fields on M Dwarfs. Astrophys. J. Lett. 459, L95. DOI ADS

Johns-Krull, C.M., Hawley, S.L., Basri, G., Valenti, J.A.: 1997, Hamilton Echelle Spectroscopy of the 1993 March 6 Solar Flare. Astrophys. J. Supp. Series 112, 221. DOI. ADS.

Karlický, M., Brown, J.C., Conway, A.J., Penny, G.: 2000, Flare hard X-rays from neutral beams. Astron. Astrophys. 353, 729. ADS

Katsova, M.M., Boiko, A.Y., Livshits, M.A.: 1997, The gas-dynamic model of impulsive stellar flares. Astron. Astrophys. 321, 549. ADS

Kašparová, J., Varady, M., Heinzel, P., Karlický, M., Moravec, Z.: 2009, Response of optical hydrogen lines to beam heating. I. Electron beams. Astron. Astrophys. 499, 923. DOI] ADS

Kepple, P., Griem, H.R.: 1968, Improved Stark Profile Calculations for the Hydrogen Lines $\mathrm{H} \alpha, \mathrm{H} \beta, \mathrm{H} \gamma$, and $\mathrm{H} \delta$. Physical Review 173, 317. DOI ADS

Kerr, G.S., Fletcher, L.: 2014, Physical Properties of White-light Sources in the 2011 February 15 Solar Flare. Astrophys. J. 783, 98. DOI ADS

Kowalski, A.F., Cauzzi, G., Fletcher, L.: 2015, Optical Spectral Observations of a Flickering White-light Kernel in a C1 Solar Flare. Astrophys. J. 798, 107. DOI, ADS.

Kowalski, A.F., Hawley, S.L., Holtzman, J.A., Wisniewski, J.P., Hilton, E.J.: 2010, A White Light Megaflare on the dM4.5e Star YZ CMi. Astrophys. J. Lett. 714, L98. DOI ADS

Kowalski, A.F., Hawley, S.L., Holtzman, J.A., Wisniewski, J.P., Hilton, E.J.: 2012, The Multiple Continuum Components in the White-Light Flare of 16 January 2009 on the dM4.5e Star YZ CMi. Solar Phys. 277, 21. DOI ADS

Kowalski, A.F., Hawley, S.L., Wisniewski, J.P., Osten, R.A., Hilton, E.J., Holtzman, J.A., Schmidt, S.J., Davenport, J.R.A.: 2013, Time-resolved Properties and Global Trends in dMe Flares from Simultaneous Photometry and Spectra. Astrophys. J. Supp. Series 207, 15. DOI ADS

Kretzschmar, M.: 2011, The Sun as a star: observations of white-light flares. Astron. Astrophys. 530, A $84+$. DOI ADS

Krucker, S., Hudson, H.S., Jeffrey, N.L.S., Battaglia, M., Kontar, E.P., Benz, A.O., Csillaghy, A., Lin, R.P.: 2011, High-resolution Imaging of Solar Flare Ribbons and Its Implication on the Thick-target Beam Model. Astrophys. J. 739, 96. DOI. ADS.

Kunkel, W.E.: 1970, On the Spectra of Stellar Flares. Astrophys. J. 161, 503. DOI ADS

Leenaarts, J., Pereira, T.M.D., Carlsson, M., Uitenbroek, H., De Pontieu, B.: 2013, The Formation of IRIS Diagnostics. II. The Formation of the $\mathrm{Mg}$ II $\mathrm{h} \& \mathrm{k}$ Lines in the Solar Atmosphere. Astrophys. J. 772, 90. DOI ADS.

Lemke, M.: 1997, Extended VCS Stark broadening tables for hydrogen - Lyman to Brackett series. Astron. Astrophys. Suppl. 122, 285. DOI. ADS

Lin, R.P., Dennis, B.R., Hurford, G.J., Smith, D.M., Zehnder, A., Harvey, P.R., Curtis, D.W., Pankow, D., Turin, P., Bester, M., Csillaghy, A., Lewis, M., Madden, N., van Beek, H.F., Appleby, M., Raudorf, T., McTiernan, J., Ramaty, R., Schmahl, E., Schwartz, R., Krucker, S., Abiad, R., Quinn, T., Berg, P., Hashii, M., Sterling, R., Jackson, R., Pratt, R., Campbell, R.D., Malone, D., Landis, D., Barrington-Leigh, C.P., Slassi-Sennou, S., Cork, C., Clark, D., Amato, D., Orwig, L., Boyle, R., Banks, I.S., Shirey, K., Tolbert, A.K., Zarro, D., Snow, F., Thomsen, K., Henneck, R., McHedlishvili, A., Ming, P., Fivian, M., Jordan, J., Wanner, R., Crubb, J., Preble, J., Matranga, M., Benz, A., Hudson, H., Canfield, R.C., Holman, G.D., Crannell, C., Kosugi, T., Emslie, A.G., Vilmer, N., Brown, J.C., Johns-Krull, C., Aschwanden, M., Metcalf, T., Conway, A.: 2002, The Reuven Ramaty High-Energy Solar Spectroscopic Imager (RHESSI). Solar Phys. 210, 3. DOI ADS 
Livshits, M.A., Badalian, O.G., Kosovichev, A.G., Katsova, M.M.: 1981, The optical continuum of solar and stellar flares. Solar Phys. 73, 269. DOI ADS

Longcope, D.W., Guidoni, S.E.: 2011, A Model for the Origin of High Density in Looptop X-Ray Sources. Astrophys. J. 740, 73. DOI ADS

Magain, P.: 1986, Contribution functions and the depths of formation of spectral lines. Astron. Astrophys. 163, 135. ADS

Mathioudakis, M., Bloomfield, D.S., Jess, D.B., Dhillon, V.S., Marsh, T.R.: 2006, The periodic variations of a white-light flare observed with ULTRACAM. Astron. Astrophys. 456, 323. DOI ADS

Mihalas, D.: 1978, Stellar atmospheres, 2nd edn. W.H. Freeman and Co., San Francisco. ADS.

Milligan, R.O., Kerr, G.S., Dennis, B.R., Hudson, H.S., Fletcher, L., Allred, J.C., Chamberlin, P.C., Ireland, J., Mathioudakis, M., Keenan, F.P.: 2014, The Radiated Energy Budget of Chromospheric Plasma in a Major Solar Flare Deduced from Multi-wavelength Observations. Astrophys. J. 793, 70. DOI ADS

Mott, N.F., Massey, H.S.W.: 1965, Theory of Atomic Collisions, 3rd edn. Oxford Univ. Press, Oxford. Chap. Xvi.

Mullan, D.J., Mathioudakis, M., Bloomfield, D.S., Christian, D.J.: 2006, A Comparative Study of Flaring Loops in Active Stars. Astrophys. J. Supp. Series 164, 173. DOI. ADS

Nayfonov, A., Däppen, W., Hummer, D.G., Mihalas, D.: 1999, The MHD Equation of State with Post-Holtsmark Microfield Distributions. Astrophys. J. 526, 451. DOI. ADS

Neidig, D.F.: 1983, Spectral analysis of the optical continuum in the 24 April 1981 flare. Solar Phys. 85, 285. DOI ADS

Neidig, D.F., Grosser, H., Hrovat, M.: 1994, Optical output of the 24 April 1984 white-light flare. Solar Phys. 155, 199. DOI ADS

Osten, R.A., Hawley, S.L., Allred, J., Johns-Krull, C.M., Brown, A., Harper, G.M.: 2006, From Radio to X-Ray: The Quiescent Atmosphere of the dMe Flare Star EV Lacertae. Astrophys. J. 647, 1349. DOI ADS

Osten, R.A., Drake, S., Tueller, J., Cummings, J., Perri, M., Moretti, A., Covino, S.: 2007, Nonthermal Hard X-Ray Emission and Iron K $\alpha$ Emission from a Superflare on II Pegasi. Astrophys. J. 654, 1052. DOI ADS.

Osten, R.A., Godet, O., Drake, S., Tueller, J., Cummings, J., Krimm, H., Pye, J., Pal'shin, V., Golenetskii, S., Reale, F., Oates, S.R., Page, M.J., Melandri, A.: 2010, The Mouse That Roared: A Superflare from the dMe Flare Star EV Lac Detected by Swift and Konus-Wind. Astrophys. J. 721, 785. DOI ADS

Paulson, D.B., Allred, J.C., Anderson, R.B., Hawley, S.L., Cochran, W.D., Yelda, S.: 2006, Optical Spectroscopy of a Flare on Barnard's Star. Pub. Astron. Soc. Pac. 118, 227. DOI ADS

Phan-Bao, N., Lim, J., Donati, J.-F., Johns-Krull, C.M., Martín, E.L.: 2009, Magnetic Field Topology in Low-Mass Stars: Spectropolarimetric Observations of M Dwarfs. Astrophys. J. 704, 1721. DOI ADS

Pillet, P., van Linden van den Heuvell, H.B., Smith, W.W., Kachru, R., Tran, N.H., Gallagher, T.F.: 1984, Microwave ionization of Na Rydberg atoms. Phys. Rev. A 30, 280. DOI ADS

Qiu, J., Liu, W., Hill, N., Kazachenko, M.: 2010, Reconnection and Energetics in Two-ribbon Flares: A Revisit of the Bastille-day Flare. Astrophys. J. 725, 319. DOI ADS

Ricchiazzi, P.J.: 1982, A static model of chromospheric heating in solar flares. PhD thesis, California Univ., San Diego. ADS.

Ricchiazzi, P.J., Canfield, R.C.: 1983, A static model of chromospheric heating in solar flares. Astrophys. J. 272, 739. DOI ADS

Robinson, R.D., Carpenter, K.G., Percival, J.W., Bookbinder, J.A.: 1995, A Search for Microflaring Activity on dMe Flare Stars. I. Observations of the dM8e Star CN Leonis. Astrophys. J. 451, 795. DOI ADS

Rybicki, G.B., Hummer, D.G.: 1992, An accelerated lambda iteration method for multilevel radiative transfer. II - Overlapping transitions with full continuum. Astron. Astrophys. 262, 209. ADS

Scharmer, G.B.: 1981, Solutions to radiative transfer problems using approximate lambda operators. Astrophys. J. 249, 720. DOI ADS

Scharmer, G.B., Carlsson, M.: 1985, A new approach to multi-level non-LTE radiative transfer problems. Journal of Computational Physics 59, 56. DOI ADS.

Schrijver, C.J., Hudson, H.S., Murphy, R.J., Share, G.H., Tarbell, T.D.: 2006, Gamma Rays and the Evolving, Compact Structures of the 2003 October 28 X17 Flare. Astrophys. J. 650, 1184. DOI ADS 
Seaton, M.J.: 1990, Atomic data for opacity calculations. XIII - Line profiles for transitions in hydrogenic ions. Journal of Physics B Atomic Molecular Physics 23, 3255. DOI ADS

Smith, K., Güdel, M., Audard, M.: 2005, Flares observed with XMM-Newton and the VLA. Astron. Astrophys. 436, 241. DOI. ADS

Smith, R.K., Brickhouse, N.S., Liedahl, D.A., Raymond, J.C.: 2001, Collisional Plasma Models with APEC/APED: Emission-Line Diagnostics of Hydrogen-like and Helium-like Ions. Astrophys. J. Lett. 556, L91. DOI ADS

Stehle, C., Jacquemot, S.: 1993, Line shapes in hydrogen opacities. Astron. Astrophys. 271, 348. ADS

Su, Y., Holman, G.D., Dennis, B.R.: 2011, Evidence for the Full Hard X-ray Spectral Signature of Nonuniform Ionization in a Solar Flare. Astrophys. J. 731, 106. DOI ADS

Sutton, K.: 1978, Approximate line shapes for hydrogen. J. Quant. Spectros. Rad. Transf. 20, 333. DOI ADS

Svestka, Z.: 1963, Spectral analysis of the moustache-like flare of August 7, 1960. Bull. Astron. Inst. Czechoslov. 14, 234. ADS

Tremblay, P.-E., Bergeron, P.: 2009, Spectroscopic Analysis of DA White Dwarfs: Stark Broadening of Hydrogen Lines Including Nonideal Effects. Astrophys. J. 696, 1755. DOI ADS

Uitenbroek, H.: 2001, Multilevel Radiative Transfer with Partial Frequency Redistribution. Astrophys. J. 557, 389. DOI ADS

Varady, M., Karlický, M., Moravec, Z., Kašparová, J.: 2014, Modifications of thick-target model: re-acceleration of electron beams by static and stochastic electric fields. Astron. Astrophys. 563, A51. DOI ADS

Vidal, C.R., Cooper, J., Smith, E.W.: 1970, Hydrogen Stark broadening calculations with the unified classical path theory. J. Quant. Spectros. Rad. Transf. 10, 1011. DOI ADS

Vidal, C.R., Cooper, J., Smith, E.W.: 1971, Unified theory calculations of Stark broadened hydrogen lines including lower state interactions. J. Quant. Spectros. Rad. Transf. 11, 263. DOI ADS

Vidal, C.R., Cooper, J., Smith, E.W.: 1973, Hydrogen Stark-Broadening Tables. Astrophys. J. Supp. Series 25, 37. DOI ADS

Wang, L., Fang, C., Ding, M.: 2007, Small-Scale Brightenings in the UV Continuum of an M9.1 Solar Flare. Chin. J. Astron. Astrophys. 7, 721. DOI ADS

Warren, H.P.: 2006, Multithread Hydrodynamic Modeling of a Solar Flare. Astrophys. J. 637, 522. DOI ADS

Wiese, W.L., Kelleher, D.E., Paquette, D.R.: 1972, Detailed Study of the Stark Broadening of Balmer Lines in a High-Density Plasma. Phys. Rev. A 6, 1132. DOI, ADS

Worden, S.P., Schneeberger, T.J., Giampapa, M.S., Deluca, E.E., Cram, L.E.: 1984, The response of chromospheric emission lines to flares on YZ Canis Minoris. Astrophys. J. 276, 270. DOI ADS

Young, P.R., Del Zanna, G., Landi, E., Dere, K.P., Mason, H.E., Landini, M.: 2003, CHIANTIAn Atomic Database for Emission Lines. VI. Proton Rates and Other Improvements. Astrophys. J. Supp. Series 144, 135. DOI ADS

Zener, C.: 1932, Non-Adiabatic Crossing of Energy Levels. Roy. Soc. London Proc. Ser. A 137, 696. DOI ADS

Zirin, H.: 1980, Three flares with blue continuum, one with a D3 preflare shell. Astrophys. J. 235, 618. [DOI ADS

Zirin, H., Neidig, D.F.: 1981, Continuum emission in the 1980 July 1 solar flare. Astrophys. J. Lett. 248, L45. DOI ADS 